\title{
Effects of Hall currents and radiation on MHD free convective flow past a oscillating vertical plate with oscillatory plate temperature in a porous medium
}

\author{
Bhaskar Chandra Sarkar, Sanatan Das and Rabidra Nath Jana \\ Department of Applied Mathematics, \\ Vidyasagar University, Midnapore 721 102, India
}

Keywords: MHD flow, Hall currents, Grashof number, radiation parameter, Darcy number, prandtl number, frequency parameter, unsteady flow, porous medium and heat transfer.

\begin{abstract}
Combined effects of Hall currents and radiation on an unsteady MHD free convective flow past a vertical flat plate with oscillatory plate temperature in a porous medium have been studied. The governing equations are solved analytically in closed form. The results obtained are presented graphically. It is found that the Hall currents accelerate the fluid velocity components whereas the radiation has a tendency to retard the fluid velocity components. Porosity of the medium has a tendency to enhance the fluid velocity components. Hall currents reduce the amplitude of the shear stress at the plate due to the unsteady primary flow whereas they increase the amplitude of the shear stress at the plate due to the unsteady secondary flow. Further, the rate of heat transfer at the plate due to unsteady flow increases with an increase in either radiation parameter or Prandtl number or frequency parameter.
\end{abstract}

\section{Introduction}

Free convective flows in a porous medium have received much attention in recent time due to its wide applications in geothermal and oil reservoir engineering as well as other geophysical and astrophysical studies. Moreover, considerable interest has been shown in radiation interaction with convection for heat transfer in fluids. This is due to the significant role of thermal radiation in the surface heat transfer when convection heat transfer is small, particularly in free convection problems involving absorbing-emitting fluids. The effects of transversely applied magnetic field on the flow of an electrically conducting viscous fluids have been discussed widely owing to their astrophysics, geophysical and engineering applications. When the strength of the magnetic field is strong, one cannot neglect the effects of Hall current. The radiative convective flow of an electrically conducting fluid in the presence of a magnetic field is encountered in geophysical and cosmical fluid dynamics. It is also important in the solar physics involved in the sunspot development. In an ionized gas where the density is low and/or the magnetic field is very strong, the conductivity normal to the magnetic field is reduced due to the free spiraling of electrons and ions about the magnetic lines of force before suffering collisions and a current is induced in a direction normal to both the electric and the magnetic fields. This current, well known in the literature, is called the Hall currents. Due to Hall currents the electrical conductivity of the fluid becomes anisotropic and this causes the secondary flow. Hall effect is important when the Hall parameter, which is the ratio between the electron-cyclotron frequency and the electron-atom-collision frequency, is high. This happens when the magnetic field is high or when the collision frequency is low. Hall currents are of great importance in many astrophysical problems, Hall accelerator and flight MHD as well as flows of plasma in a MHD power generator. Hall currents and surface temperature oscillation effects on natural convection magnetohydrodynamic heat-generating flow have been considered by

Takhar and Ram [1]. The unsteady hydromagnetic free convection flow with Hall currents and mass transfer along an accelerated porous plate with time dependent temperature and concentration has been studied by Sattar and Hossain [2]. Alagoa et al. [3] have studied magnetohydrodynamic optically-transparent free-convection flow, with radiative heat transfer in porous media with timedependent 
suction using an asymptotic approximation, showing that thermal radiation exerts a significant effect on the flow dynamics. Kinyanjui et al.[4] have presented the magnetohydrodynamic free convection heat and mass transfer of a heat generating fluid past an impulsively started infinitely long vertical porous plate with Hall current and radiation absorption. Hayat et al. [5] have determined the Hall effects on the unsteady hydromagnetic oscillatory flow of a second-grade fluid. Comprehensive literature on various aspects of free convection flows and its applications have been discussed by Nield and Bejan [6]. The thermal radiation interaction on an absorbing emitting fluid past a vertical porous plate immersed in a porous medium has been reported by Samad and Rahman [7]. The heat and mass transfer of an unsteady MHD natural convective flow of a rotating fluid past a vertical porous flat plate in the presence of radiative heat transfer have been studied by Mbeledogu and Ogulu [8]. Beg and Ghosh [9] have investigated hydromagnetic free and forced convection of an optically-thin gray gas from vertical flat plate subject to a surface temperature oscillations with significant thermal radiation. The effects of thermal radiation, Hall currents, Soret, and Dufour on MHD flow by mixed convection over a vertical surface in porous media have been studied by Shateyi et al.[10]. Effects of radiation on free convection flow in a vertical channel embedded in porous media have been studied by Das et al. [11, 12]. Krishna and Sujatha [13] have discussed MHD free and forced convection flow of newtonian fluid through a porous medium in an infinite vertical plate in presence of thermal radiation heat transfer and surface temperature oscillation..

The aim of our present paper is to study the Hall effects on MHD free convection flow of an electrically conducting, newtonian, optically-thin fluid past a vertical flat plate with thermal radiation and oscillating plate temperature in a porous medium. It is found that thermal radiation retards the steady as well as the unsteady fluid velocity components whereas the buoyancy force and Hall currents accelerate the fluid velocity components. The frequency parameter leads to rise the unsteady fluid velocity components. The fluid temperature falls due to an increase in radiation parameter for both the steady as well as unsteady fluid velocity components. The fluid temperature decreases with an increase in either Prandtl number or phase angle whereas it increases with an increase in frequency parameter. In the steady flow, the absolute value of the shear stress at the plate $(\eta=0)$ due to the primary flow decreases whereas the shear stress at the plate $(\eta=0)$ due to the secondary flow increases with an increase in Hall parameter $m$. Further, the amplitude of the rate of heat transfer at the plate due to unsteady flow increases with an increase in frequency parameter $n$. In the absence of Hall current $(m=0)$, the steady and unsteady temperature distributions and the fluid velocity components do not identical with the results obtained by Krishna and Sujatha [13]. This is due to the error in the mathematical formulation of their problem. It is noted that the boundary condition for $w_{0}$ and $w_{1}$ in the equations (2.35) and (2.37) of Krishna and Sujatha [13] suggest that $w_{0}(y)=0$ and $w_{1}(y)=0$ everywhere in the flow field. The forms $u_{0}+i w_{0}=F$ and $u_{1}+i w_{1}=H$ given by equations (2.30) and 2.31) of Krishna and Sujatha [13] are meaningless. The graphs of $w_{1}$ in Figs.10,11, 12, 13 and 14 are contradictory with the mathematical expression of $w_{1}\left(\right.$ as $\left.w_{1}=0\right)$.

\section{Formulation of the problem and its solutions}

Consider an unsteady MHD flow of a viscous incompressible electrically conducting fluid past an infinitely long vertical flat plate embedded in a porous medium on taking Hall current into account. Choose a Cartesian co-ordinates system with $x$-axis along the plate in the direction of the flow, the $y$-axis is normal to the plate and the $z$-axis is perpendicular to $x y$-plane(see Fig.1). The plate is moving with oscillatory velocity $U(1+\varepsilon \cos \omega t), U$ being the mean velocity, $\omega$ being the frequency of the oscillations and $\varepsilon(=1)$ is a real number. The plate temperature oscillates with the same frequency as that of the plate oscillations about a non-uniform mean temperature. A uniform magnetic field $B_{0}$ is applied transverse to the plate. Since the plate is infinitely long, all physical variables depend on $y$ and $t$ only. The fluid is considered to be gray, 
absorbing-emitting radiation but non-scattering medium. Since the magnetic Reynolds number is very small for metallic liquid or partially ionized fluid the induced magnetic field produced by the electrically conducting fluid is neglected.

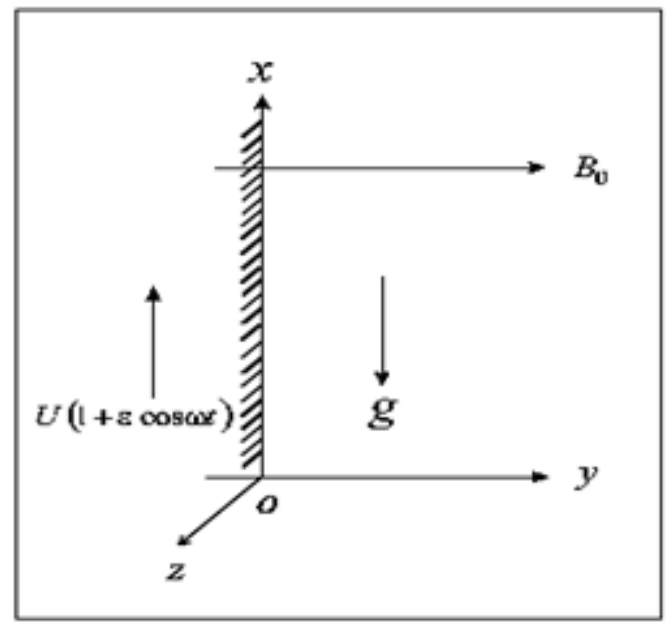

Fig. 1: Geometry of the problem

Generalized Ohm's law on taking Hall current into account is [see Cowling [14]]

$$
\bar{j}+\frac{\omega_{\varepsilon} \tau_{\varepsilon}}{B_{0}}(\bar{j} \times \bar{B})=\sigma(\bar{E}+\bar{q} \times \bar{B})
$$

Where $\bar{q}, \bar{B}, \bar{E}, \bar{j}, \sigma, \omega_{e}$ and $\tau_{e}$ are respectively the velocity vector, the magnetic field vector, the electric field vector, the current density vector, electric conductivity, cyclotron frequency and electron collision time. In writing the equation (1), the ion-slip and the thermoelectric effects as well as the electron pressure gradient are neglected.

The equation of continuity $\nabla \cdot \bar{q}=0$ with no-slip condition at the plate gives $v=0$ everywhere in the flow where $\bar{q} \equiv(u, v, w), u, v$ and $w$ are respectively velocity components along the coordinate axes. The solenoidal relation $\nabla \cdot \bar{B}=0$ gives $B_{y}=$ constant $=B_{0}$ everywhere in the flow where $\bar{B} \equiv\left(0, B_{y}, 0\right)$. The conservation of electric current $\nabla \cdot \bar{j}=0$ yields $j_{y}=$ constant where $\bar{j} \equiv\left(j_{x}, j_{y}, j_{z}\right)$. This constant is zero since $j_{y}=0$ at the plate which is electrically non-conducting. Hence, $j_{y}=0$ everywhere in the flow. As the induced magnetic field is neglected, the Maxwell's equation $\nabla \times \bar{E}=-\frac{\partial \bar{B}}{\partial t}$ becomes $\nabla \times \bar{E}=0$ which gives $\frac{\partial E_{x}}{\partial y}=0$ and $\frac{\partial E_{z}}{\partial y}=0$. This implies that $E_{x}=$ constant and $E_{z}=$ constant everywhere in the flow.

In view of the above assumption, equation (1) yields

$$
\begin{gathered}
j_{x}-m j_{z}=\sigma\left(E_{x}-w B_{0}\right), \\
j_{z}+m j_{x}=\sigma\left(E_{z}+u B_{0}\right),
\end{gathered}
$$

where $m=\omega_{\varepsilon} \tau_{\varepsilon}$ is the Hall parameter.

As $y \rightarrow \infty, j_{x} \rightarrow 0, j_{y} \rightarrow 0$, since the magnetic field is uniform at infinity. Also, $u \rightarrow 0, w \rightarrow 0$ as $y \rightarrow \infty$. Using these conditions, equations (2) and (3) give $E_{x}=0$ and $E_{y}=0$ everywhere in the flow.

Solving for $j_{x}$ and $j_{z}$ from (2) and (3), on taking $E_{x}=E_{y}=0$, we have 


$$
\begin{aligned}
& j_{x}=\frac{\sigma B_{0}}{1+m^{2}}(m u-w), \\
& j_{z}=\frac{\sigma B_{0}}{1+m^{2}}(m w+u) .
\end{aligned}
$$

On the use of (4) and (5), the equations of motion are

$$
\begin{aligned}
& \frac{\partial u}{\partial t}=v \frac{\partial^{2} u}{\partial y^{2}}+g \beta^{*}\left(T-T_{\infty}\right)-\frac{\sigma B_{0}{ }^{2}}{\rho\left(1+m^{2}\right)}(m w+u)-\frac{v}{k_{1}} u, \\
& \frac{\partial w}{\partial t}=v \frac{\partial^{2} w}{\partial y^{2}}-\frac{\sigma B_{0}{ }^{2}}{\rho\left(1+m^{2}\right)}(w-m u)-\frac{v}{k_{1}} w .
\end{aligned}
$$

Where $k_{1}$ is the permeability of the porous medium, $\nu$ the kinematic coefficient of viscosity $\beta$ the coefficient of thermal expansion, $t$ time, $g$ the gravitational acceleration and $\rho$ the fluid density. The energy equation is

$$
\frac{\partial T}{\partial t}=\frac{k}{\rho c_{p}} \frac{\partial^{2} T}{\partial y^{2}}-\frac{1}{\rho c_{p}} \frac{\partial q_{r}}{\partial y},
$$

where $T$ is the temperature of the fluid, $q_{r}$ the radiative heat flux, $k$ the thermal conductivity and $c_{p}$ the specific heat at constant pressure. For the case of an optically-thin gray gas, the thermal radiation flux gradient may be expressed according to Siegel and Howell [15] as

$$
-\frac{\partial q_{r}}{\partial y}=4 k^{*} \sigma^{*}\left(T_{\infty}^{4}-T^{4}\right)
$$

where $T_{\infty}$ is the temperature far away from the plate $k$ the absorption coefficient of the fluid and $\sigma$ the Stefan-Boltzmann constant. It is assumed that the temperature differences within the flow are sufficiently small such that $T^{4}$ may be expressed as a linea function of temperature. This is accomplished by expanding $T^{4}$ in a Taylor series about $T_{\infty}$ and neglecting higher-order terms, we get

$$
T^{4} \cong 4 T_{\infty}^{3} T-3 T_{\infty}^{4} \text {. }
$$

On the use of equations (9) and (10), equation (8) becomes

$$
\frac{\rho c_{p}}{k} \frac{\partial T}{\partial t}=\frac{\partial^{2} T}{\partial y^{2}}-\frac{16 k^{*} \sigma^{*} T_{\infty}^{3}\left(T-T_{\infty}\right)}{k} \text {. }
$$

The boundary conditions for the velocity field and the temperature distribution are

$$
\begin{aligned}
& u=U(1+\varepsilon \cos \omega t), w=0, T=T_{w}+\varepsilon\left(T_{w}-T_{\infty}\right) \cos \omega t \text { at } y=0, \\
& u \rightarrow 0, w \rightarrow 0, T \rightarrow T_{\infty} \text { as } y \rightarrow \infty,
\end{aligned}
$$

where $T_{w}$ is the plate temperature.

Introducing the non-dimensional variables

$$
\eta=\frac{y U}{v},\left(u_{1}, w_{1}\right)=\frac{(u, w)}{U}, \tau=\frac{t U^{2}}{v}, \theta=\frac{T-T_{\infty}}{T_{w}-T_{\infty}},
$$

equations (6), (7) and (8) become

$$
\begin{aligned}
& \frac{\partial u_{1}}{\partial \tau}=\frac{\partial^{2} u_{1}}{\partial \eta^{2}}+G r \theta-\frac{M^{2}}{1+m^{2}}\left(m w_{1}+u_{1}\right)-\frac{u_{1}}{D a}, \\
& \frac{\partial w_{1}}{\partial \tau}=\frac{\partial^{2} w_{1}}{\partial \eta^{2}}-\frac{M^{2}}{1+m^{2}}\left(w_{1}-m u_{1}\right)-\frac{w_{1}}{D a}, \\
& \operatorname{Pr} \frac{\partial \theta}{\partial \tau}=\frac{\partial^{2} \theta}{\partial \eta^{2}}-\operatorname{Ra} \theta,
\end{aligned}
$$


where $M^{2}=\frac{\sigma B_{0}^{2} v}{\rho U^{2}}$ is the magnetic parameter, $G r=\frac{g \beta^{*}\left(T_{w}-T_{\infty}\right) v}{U^{3}}$ the Grashof number, $D a=\frac{k_{1} U^{2}}{v^{2}}$ the Darcy number, $\operatorname{Pr}=\frac{\rho c_{p} v}{k}$ the Prandtl number and $R a=\frac{16 k^{*} \sigma^{*} T_{\infty}^{3} v^{2}}{k U^{2}}$ the radiation parameter.

The boundary conditions (12) become

$$
\begin{aligned}
& u_{1}=1+\varepsilon \cos n \tau, w_{1}=0, \theta=1+\varepsilon \cos n \tau \text { at } \eta=0, \\
& u_{1} \rightarrow 0, w_{1} \rightarrow 0, \theta \rightarrow 0 \text { as } \eta \rightarrow \infty,
\end{aligned}
$$

where $n=\frac{v \omega}{U^{2}}$ is the frequency parameter.

Combining equations (14) and (15), we get

$$
\frac{\partial f}{\partial \tau}=\frac{\partial^{2} f}{\partial \eta^{2}}+G r \theta-\left[\frac{M^{2}}{1+m^{2}}(1-i m)+\frac{1}{D a}\right] f,
$$

where

$$
f=u_{1}+i w_{1} \text { and } i=\sqrt{-1} .
$$

The corresponding boundary conditions for $f$ and $\theta$ are

$$
\begin{aligned}
& f=1+\varepsilon \cos n \tau, \quad \theta=1+\varepsilon \cos n \tau \text { at } \eta=0, \\
& f \rightarrow 0, \theta \rightarrow 0 \text { as } \eta \rightarrow \infty .
\end{aligned}
$$

To solve the differential equations (18) and (16), we assume

$$
\begin{aligned}
& f(\eta, \tau)=f_{0}(\eta)+\varepsilon\left[f_{1}(\eta) e^{i n t}+f_{2}(\eta) e^{-i n t}\right] \\
& \theta(\eta, \tau)=\theta_{0}(\eta)+\varepsilon\left[\theta_{1}(\eta) e^{i n t}+\theta_{2}(\eta) e^{-i n t}\right]
\end{aligned}
$$

Where $f_{0}$ and $\theta_{0}$ represent respectively the steady state velocity and temperature.

On the use of (21) and (22), equations (18) and (16) give,

$$
\begin{aligned}
& \frac{d^{2} f_{0}}{d \eta^{2}}-\left[\frac{M^{2}}{1+m^{2}}(1-i m)+\frac{1}{D a}\right] f_{0}+G r \theta_{0}=0, \\
& \frac{d^{2} f_{1}}{d \eta^{2}}-\left[\frac{M^{2}}{1+m^{2}}(1-i m)+\frac{1}{D a}\right] f_{1}+G r \theta_{1}=i n f_{1}, \\
& \frac{d^{2} f_{2}}{d \eta^{2}}-\left[\frac{M^{2}}{1+m^{2}}(1-i m)+\frac{1}{D a}\right] f_{2}+G r \theta_{2}=-i n f_{2}, \\
& \frac{d^{2} \theta_{0}}{d \eta^{2}}-\operatorname{Ra} \theta_{0}=0, \\
& \frac{d^{2} \theta_{1}}{d \eta^{2}}-(\operatorname{Ra}+i n \operatorname{Pr}) \theta_{1}=0, \\
& \frac{d^{2} \theta_{2}}{d \eta^{2}}-(\operatorname{Ra}-i n \operatorname{Pr}) \theta_{2}=0 .
\end{aligned}
$$

The corresponding boundary conditions for $f_{0}, f_{1}, f_{2}, \theta_{0}, \theta_{1}$ and $\theta_{2}$ are

$$
\begin{aligned}
& f_{0}=1, \theta_{0}=1, f_{1}=\frac{1}{2}, \theta_{1}=\frac{1}{2}, f_{2}=\frac{1}{2}, \theta_{2}=\frac{1}{2} \text { at } \eta=0, \\
& f_{0} \rightarrow 0, \theta_{0} \rightarrow 0, f_{1} \rightarrow 0, \theta_{1} \rightarrow 0, f_{2} \rightarrow 0, \theta_{2} \rightarrow 0 \text { as } \eta \rightarrow \infty .
\end{aligned}
$$


The solutions of the equations (23) - (28) subject to the boundary conditions (29) are

$$
\begin{aligned}
& \theta_{0}=e^{-\sqrt{R u} \eta}, \\
& \theta_{1}=\frac{1}{2} e^{-(\alpha+i \beta) \eta}, \\
& \theta_{2}=\frac{1}{2} e^{-(\alpha-i \beta) \eta}, \\
& f_{0}=e^{-\left(\alpha_{1}-i \beta_{1}\right) \eta}-a_{1}\left(e^{-\sqrt{R a} \eta}-e^{-\left(\alpha_{1}-i \beta_{1}\right) \eta}\right), \\
& f_{1}=\frac{1}{2} e^{-\left(\alpha_{2} \pm i \beta_{2}\right) \eta}-a_{2}\left(e^{-(\alpha+i \beta) \eta}-e^{-\left(\alpha_{2} \pm i \beta_{2}\right) \eta}\right), \\
& f_{2}=\frac{1}{2} e^{-\left(\alpha_{3}-i \beta_{3}\right) \eta}-a_{3}\left(e^{-(\alpha-i \beta) \eta}-e^{-\left(\alpha_{3}-i \beta_{3}\right) \eta}\right)
\end{aligned}
$$

Where

$$
\begin{aligned}
& \alpha, \beta=\frac{1}{\sqrt{2}}\left[\left(R a^{2}+n^{2} P r^{2}\right)^{\frac{1}{2}} \pm R a\right]^{\frac{1}{2}}, \\
& \left.\alpha_{1}, \beta_{1}=\sqrt{\frac{M^{2} D a+\left(1+m^{2}\right)}{2 D a\left(1+m^{2}\right)}\left[\left\{1+\left(\frac{m D a M^{2}}{M^{2} D a+\left(1+m^{2}\right)}\right)^{2}\right\}^{\frac{1}{2}} \pm 1\right.}\right]^{\frac{1}{2}}, \\
& \left.\alpha_{2}, \beta_{2}=\sqrt{\frac{M^{2} D a+\left(1+m^{2}\right)}{2 D a\left(1+m^{2}\right)}\left[\left\{1+\left(\frac{n D a\left(1+m^{2}\right)-m D a M^{2}}{M^{2} D a+\left(1+m^{2}\right)}\right)^{2}\right\}^{\frac{1}{2}} \pm 1\right.}\right]^{\frac{1}{2}} \text {, }
\end{aligned}
$$

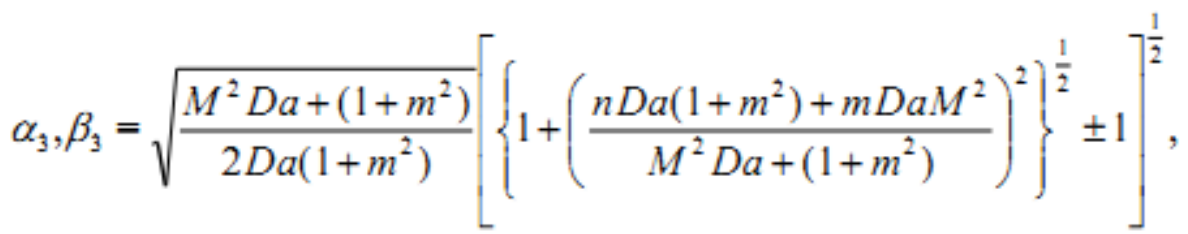

$$
\begin{aligned}
& a_{1}=\frac{G r}{\left[R a-\left(\frac{M^{2}}{1+m^{2}}+\frac{1}{D a}\right)\right]+i \frac{m M^{2}}{1+m^{2}}}, \\
& a_{2}=\frac{1}{2} \frac{G r}{\left.\operatorname{Ra}-\left(\frac{M^{2}}{1+m^{2}}+\frac{1}{D a}\right)\right]+i\left[n(\operatorname{Pr}-1)+\frac{m M^{2}}{1+m^{2}}\right]} \text {, } \\
& a_{3}=\frac{1}{2} \frac{G r}{\left.\operatorname{Ra} a-\left(\frac{M^{2}}{1+m^{2}}+\frac{1}{D a}\right)\right]-i\left[n(\operatorname{Pr}-1)-\frac{m M^{2}}{1+m^{2}}\right]} \text {. } \\
& n>\frac{m M^{2}}{1+m^{2}} \text { and lower sign for } n<\frac{m M^{2}}{1+m^{2}} \\
& \text { For the steady state, the temperature distribution and velocity components are given by } \\
& \theta_{s}=e^{-\sqrt{R a} \eta} \text {, } \\
& u_{s}=e^{-\alpha_{1} \eta}\left\{\left(1+x_{1}\right) \cos \beta_{1} \eta+y_{1} \sin \beta_{1} \eta\right\}-x_{1} e^{-\sqrt{R_{s}} \eta},
\end{aligned}
$$




$$
w_{s}=y_{1} e^{-\sqrt{R_{a}} \eta}+e^{-\alpha_{1} \eta}\left\{\left(1+x_{1}\right) \sin \beta_{1} \eta-y_{1} \cos \beta_{1} \eta\right\},
$$

Where

$$
x_{1}=\frac{G r\left[R a-\left(\frac{M^{2}}{1+m^{2}}+\frac{1}{D a}\right)\right]}{\left[\operatorname{Ra}-\left(\frac{M^{2}}{1+m^{2}}+\frac{1}{D a}\right)\right]^{2}+\left[\frac{m M^{2}}{1+m^{2}}\right]^{2}}, y_{1}=\frac{G r \frac{m M^{2}}{\left(1+m^{2}\right)}}{\left[\operatorname{Ra}-\left(\frac{M^{2}}{1+m^{2}}+\frac{1}{D a}\right)\right]^{2}+\left[\frac{m M^{2}}{1+m^{2}}\right]^{2}},
$$

and $\alpha_{1}$ and $\beta_{1}$ are given by (36) and (.) stands for steady state solution.

The unsteady temperature distribution and velocity components are

$$
\begin{aligned}
& \theta_{u s}=e^{-\alpha \eta} \cos (n \tau-\beta \eta), \\
& u_{u s}=e^{-\alpha_{2} \eta}\left\{\left(\frac{1}{2}+x_{2}\right) \cos \left(n \tau \mp \beta_{2} \eta\right)+y_{2} \sin \left(n \tau \mp \beta_{2} \eta\right)\right\} \\
& +e^{-\alpha_{3} \eta}\left\{\left(\frac{1}{2}+x_{3}\right) \cos \left(n \tau-\beta_{3} \eta\right)+y_{3} \sin \left(n \tau-\beta_{3} \eta\right)\right\} \\
& -e^{-\alpha \eta}\left\{\left(x_{2}+x_{3}\right) \cos (n \tau-\beta \eta)+\left(y_{2}+y_{3}\right) \sin (n \tau-\beta \eta)\right\}, \\
& w_{u s}=e^{-\alpha \alpha_{2} \eta}\left\{\left(\frac{1}{2}+x_{2}\right) \sin \left(n \tau \mp \beta_{2} \eta\right)-y_{2} \cos \left(n \tau \mp \beta_{2} \eta\right)\right\} \\
& -e^{-\alpha_{3} \eta}\left\{\left(\frac{1}{2}+x_{3}\right) \sin \left(n \tau-\beta_{3} \eta\right)-y_{3} \cos \left(n \tau-\beta_{3} \eta\right)\right\} \\
& -e^{-\alpha \eta \eta}\left\{\left(x_{2}-x_{3}\right) \sin (n \tau-\beta \eta)-\left(y_{2}-y_{3}\right) \cos (n \tau-\beta \eta)\right\},
\end{aligned}
$$

where

$$
\begin{aligned}
& x_{2}=\frac{1}{2} \frac{G r\left[\operatorname{Ra}-\left(\frac{M^{2}}{1+m^{2}}+\frac{1}{D a}\right)\right]}{\left.\operatorname{Ra}-\left(\frac{M^{2}}{1+m^{2}}+\frac{1}{D a}\right)\right]^{2}+\left[n(\operatorname{Pr}-1)+\frac{m M^{2}}{1+m^{2}}\right]^{2}}, \\
& y_{2}=\frac{1}{2} \frac{G r\left[n(\operatorname{Pr}-1)+\frac{m M^{2}}{1+m^{2}}\right]}{\left[\operatorname{Ra}-\left(\frac{M^{2}}{1+m^{2}}+\frac{1}{D a}\right)\right]^{2}+\left[n(P r-1)+\frac{m^{2}}{1+m^{2}}\right]^{2}}, \\
& x_{3}=\frac{1}{2} \frac{G r\left[\operatorname{Ra}-\left(\frac{M^{2}}{1+m^{2}}+\frac{1}{D a}\right)\right]}{\left[\operatorname{Ra}-\left(\frac{M^{2}}{1+m^{2}}+\frac{1}{D a}\right)\right]^{2}+\left[n(P r-1)-\frac{m M^{2}}{1+m^{2}}\right]^{2}}, \\
& y_{3}=\frac{1}{2} \frac{G r\left(n(P r-1)-\frac{m M^{2}}{1+m^{2}}\right]}{\left[\operatorname{Ra}-\left(\frac{M^{2}}{1+m^{2}}+\frac{1}{D a}\right)\right]^{2}+\left[n(P r-1)-\frac{m M^{2}}{1+m^{2}}\right]^{2}},
\end{aligned}
$$

$\alpha, \beta, \alpha_{2}, \beta_{2}, \alpha_{3}, \beta_{3}$ are given by (36) and (.) us stands for unsteady state solution. 
In the absence of Hall currents $(m=0)$, the steady state temperature distribution and velocity components become

$$
\begin{gathered}
\theta_{s}=e^{-\sqrt{R a} \eta}, \\
u_{s}=e^{-\sqrt{M^{2}+\frac{1}{D a} \eta}}+\frac{G r}{R a-\left(M^{2}+\frac{1}{D a}\right)}\left(e^{-\sqrt{M^{2}+\frac{1}{D a}} \eta}-e^{-\sqrt{R a} \eta}\right), \\
w_{s}=0,
\end{gathered}
$$

The unsteady state temperature distribution and velocity components are

$$
\begin{aligned}
& \theta_{u s}=e^{-\alpha \eta} \cos (n \tau-\beta \eta), \\
& u_{u s}=2 e^{-\alpha \alpha_{2} \eta}\left\{\left(\frac{1}{2}+x_{2}\right) \cos \left(n \tau-\beta_{2} \eta\right)+y_{2} \sin \left(n \tau-\beta_{2} \eta\right)\right\} \\
& -2 e^{-\alpha \eta}\left\{x_{2} \cos (n \tau-\beta \eta)+y_{2} \sin (n \tau-\beta \eta)\right\}, \\
& w_{u s}=0,
\end{aligned}
$$

Where

$$
\begin{aligned}
& x_{2}=\frac{1}{2} \frac{G r\left[\operatorname{Ra}-\left(M^{2}+\frac{1}{D a}\right)\right]}{\left.\operatorname{Ra}-\left(M^{2}+\frac{1}{D a}\right)\right]^{2}+n^{2}(P r-1)^{2}}, \\
& y_{2}=\frac{1}{2} \frac{G r n(P r-1)}{\left[\operatorname{Ra}-\left(M^{2}+\frac{1}{D a}\right)\right]^{2}+n^{2}(P r-1)^{2}}, \\
& \alpha, \beta=\frac{1}{\sqrt{2}\left[\left(R a^{2}+n^{2} \operatorname{Pr}^{2}\right)^{\frac{1}{2}} \pm R a\right]^{\frac{1}{2}},}[]^{\frac{M^{2} D a+1}{2 D a}}\left[\left\{1+\left(\frac{n D a}{M^{2} D a+1}\right)^{2}\right\}^{\frac{1}{2}} \pm 1\right]^{\frac{1}{2}} .
\end{aligned}
$$

Equation (46) contradicts the results obtained by Krishna and Sujatha [13] [see equation (2.43) of Krishna and Sujatha [13]]. Further, the expressions of $C_{1}, D_{1}, C_{2}$ and $D_{2}$ are not given by Krishna and Sujatha [13].

\section{Results and discussion}

We have presented the non-dimensional velocity components and temperature distribution against $\eta$ for several values of magnetic parameter $M^{2}$, Grashof number $G r$, Hall parameter $m$, radiation parameter $R a$, Darcy number $D a$, Prandtl number $P r$, frequency parameter $n$ and phase angle $n \tau$ in Figs.2-19. Figs.2-6 correspond to the steady primary velocity and the secondary velocity for which there will be no surface temperature oscillations i.e. $(n=0)$. It is seen from Fig.2 that the steady primary velocity $u_{S}$ decreases while the steady secondary velocity $w_{S}$ increases near the plate $(\eta=0)$ with an increase in magnetic parameter $M^{2}$. This implies that 
magnetic field has a retarding influence on the steady primary velocity whereas it has an accelerating influence on the steady secondary velocity. Fig.3 shows that both the steady primary velocity $u_{s}$ and the steady secondary velocity $w_{s}$ increase in the vicinity of the plate with an increase in Grashof number $G r$ which implies that the buoyancy force boosts the steady flow. It is revealed from Fig.4 that both the steady primary velocity $u_{s}$ as well as the steady secondary velocity $w_{s}$ increase near the plate with an increase in Hall parameter $m$. This indicates that Hall currents have a tendency to accelerate the fluid flows. The radiation parameter $R a$ represents the relative contribution of thermal radiative heat transfer to thermal conduction heat transfer. For $R a<1$, thermal conduction exceeds thermal radiation and for $R a>1$, this situation is reversed. For $R a>1$, this situation is reversed. For $R a=1$, the contribution from both modes is equal. Fig.5 shows that as $R a$ increases the steady flow velocities $u_{s}$ as well as $w_{s}$ decreases smoothly. It is revealed from Fig. 6 that both the steady primary velocity $u_{s}$ as well as the steady secondary velocity $w_{s}$ near the plate increase with an increase in Darcy number $D a$.

Darcy number is the measurement of the porosity of the medium. As the porosity of the medium increases, the value of $D a$ increases. For large porosity of the medium fluid gets more space to flow as a consequence its velocity increases. Figs.7-14 display the unsteady primary and the secondary velocities. It is observed from Fig.7 that both the unsteady primary velocity $u_{u s}$ and the unsteady secondary velocity $w_{u s}$ decrease with an increase in $M^{2}$. The presence of a magnetic field normal to the flow in an electrically conducting fluid introduces a Lorentz force which acts against the flow. This resistive force tends to slow down the flows and hence the fluid velocity decrease with an increase in magnetic parameter. This trend is consistent with many classical studies on magneto-convection flow. Figs.8, 9 and 11 show that both the unsteady primary velocity $u_{u s}$ as well as the unsteady secondary velocity $w_{u s}$ increase near the plate with an increase in either Grashof number $G r$ or Hall parameter $m$ or Darcy number $D a$. This indicates that the buoyancy force, Hall currents and the porosity of the medium have an accelerating influence on the fluid flows. It is seen from Figs.10, 12 and 14 that both the unsteady primary velocity $u_{u s}$ as well as the unsteady secondary velocity $w_{u s}$ decrease near the plate surface with an increase in either the radiation parameter $R a$ or Prandtl number $P r$ or phase angle $n \tau$. Physically, this is true because the increase in the Prandtl number is due to increase in the viscosity of the fluid which makes the fluid thick and hence causes a decrease in the velocity of the fluid. Fig.13 displays that an increase in frequency parameter $n$ leads to rise in the fluid flows which means that the momentum diffusivity of the fluid increases as compared to its thermal diffusivity. 


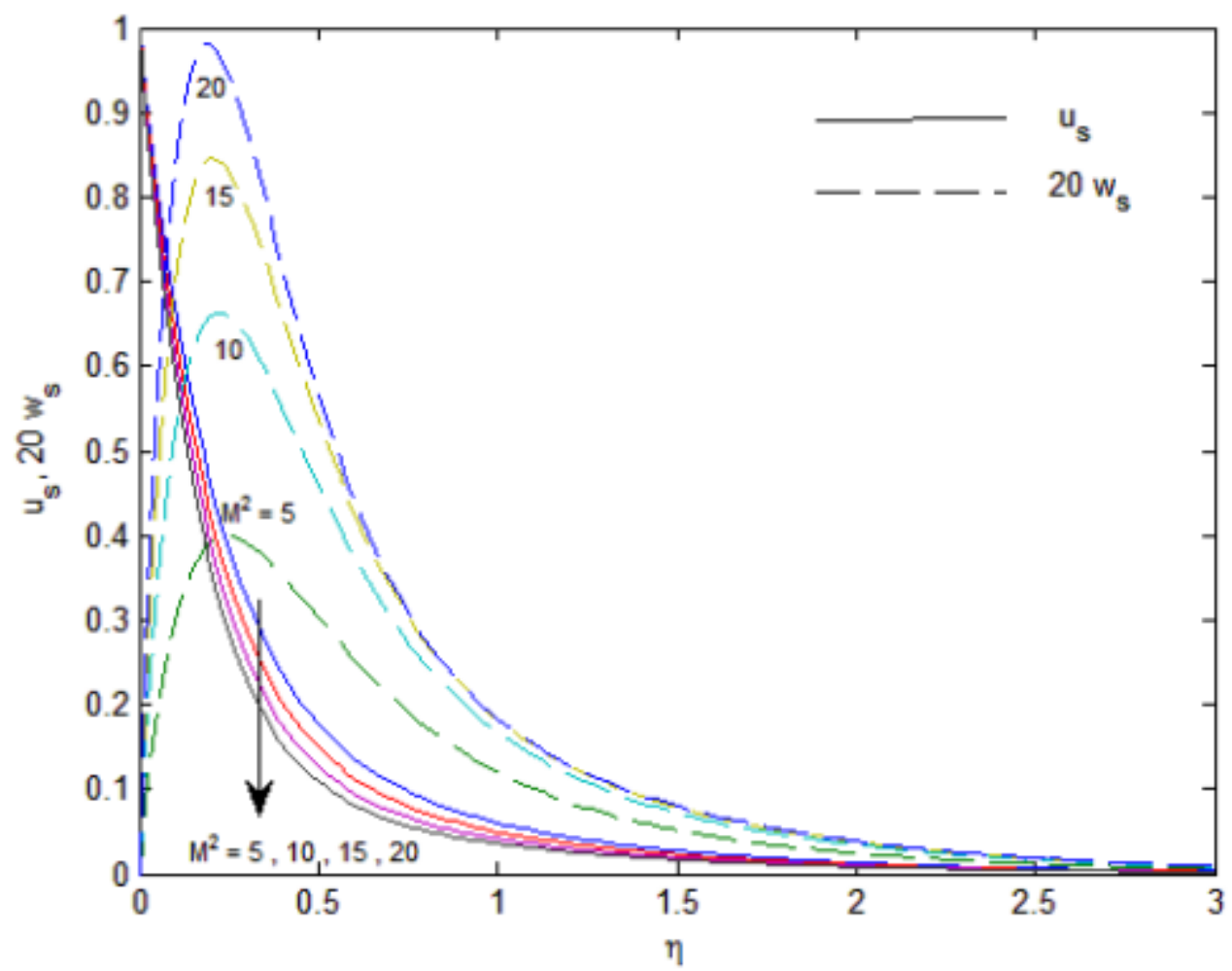

Fig. 2: Steady state velocities for different $M^{2}$ when $m=0.5, G r=5, D a=0.05$ and $R a=2$

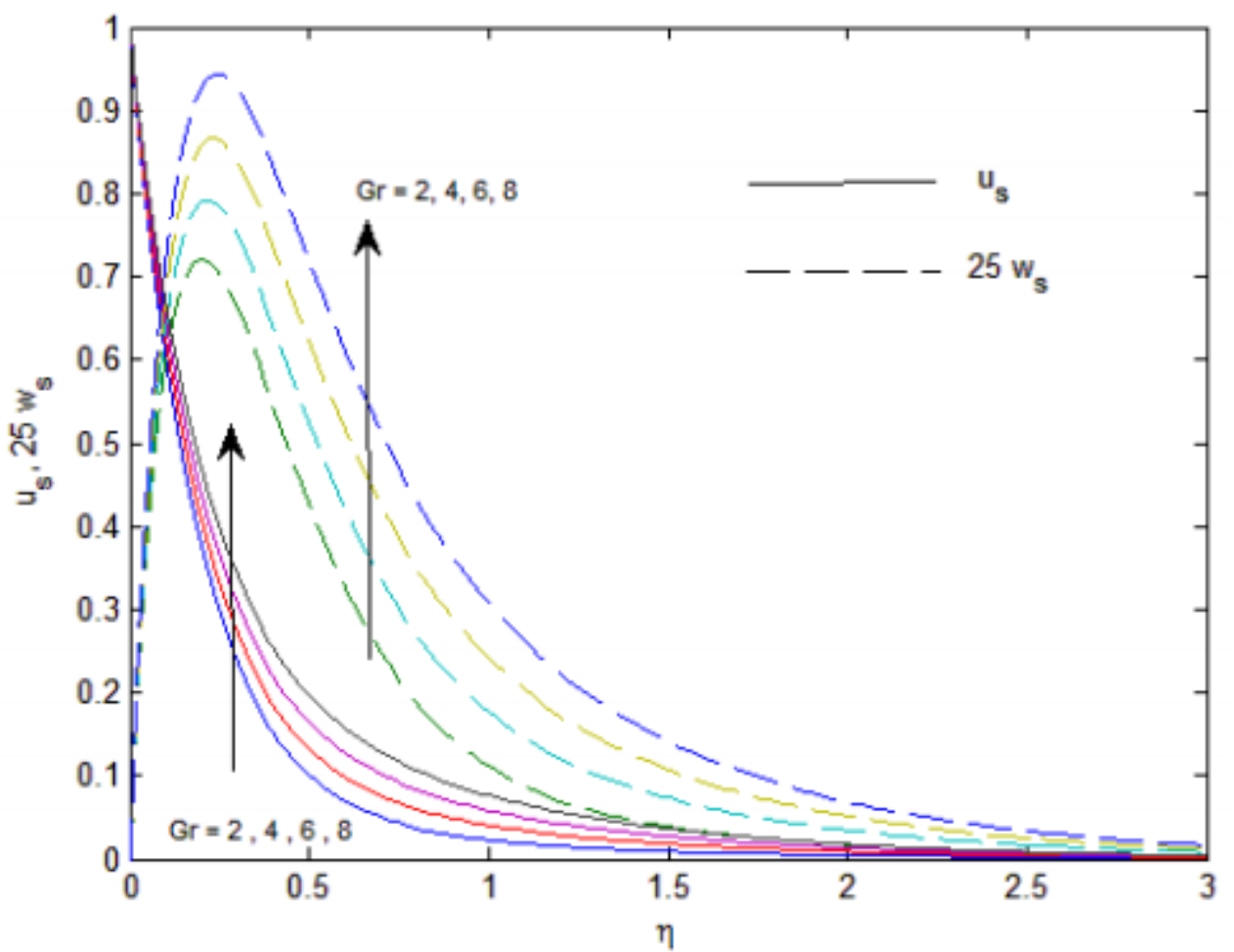

Fig. 3: Steady state velocities for different $G r$ when $m=0.5, M^{2}=10 D a=0.05$ and $R a=2$ 


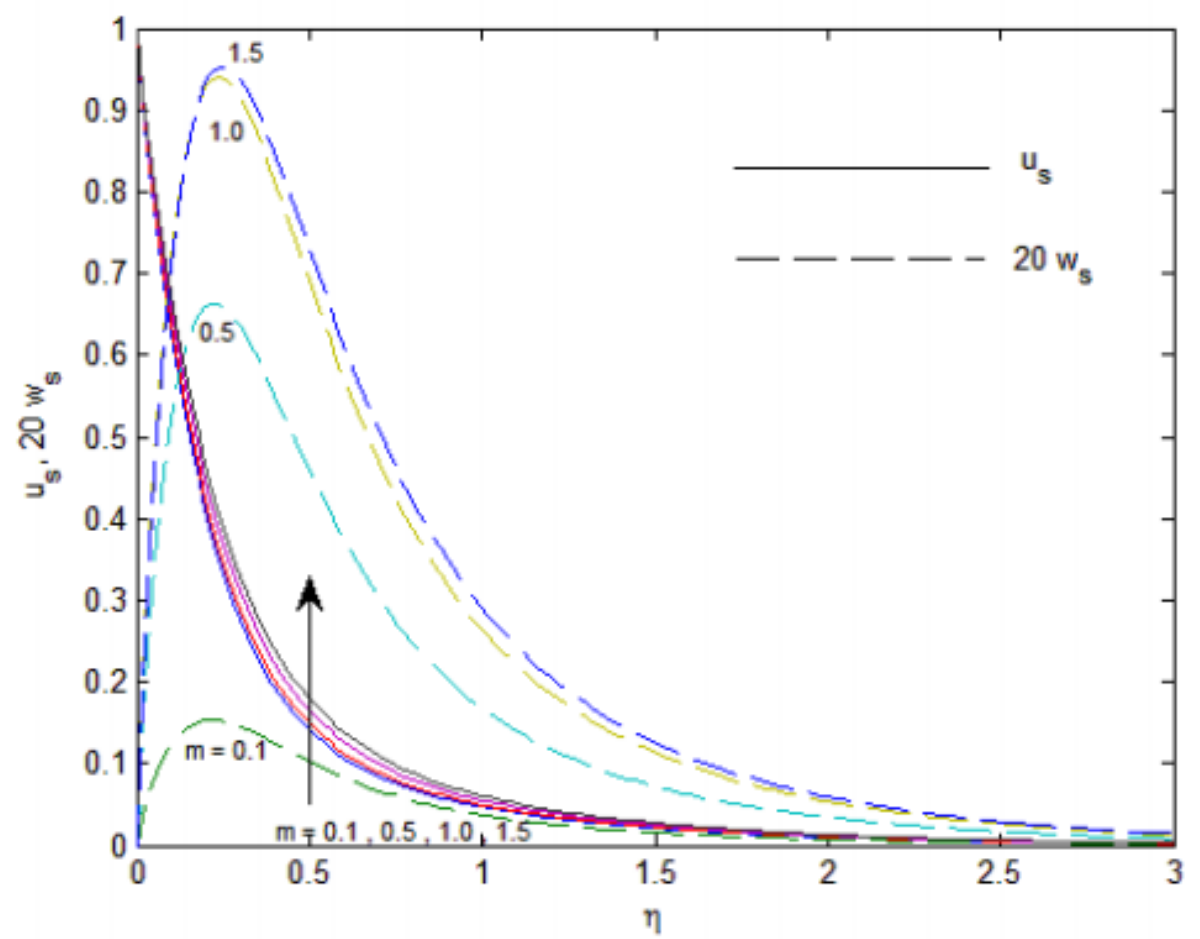

Fig. 4: Steady state velocities for different $m$ when $M^{2}=10, G r=5, D a=0.05$ and $R a=2$

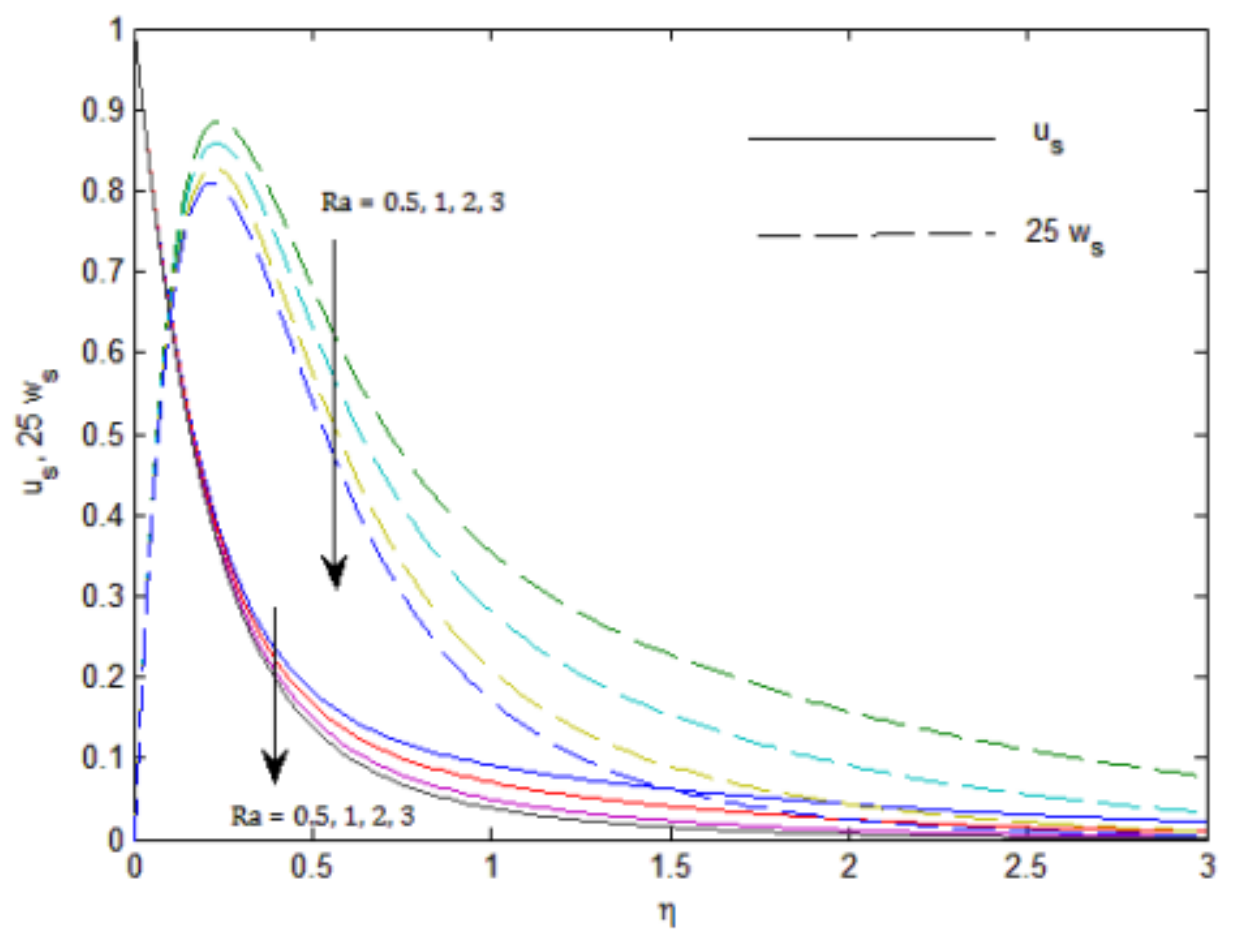

Fig. 5: Steady state velocities for different $R a$ when $m=0.5, G r=5, D a=0.05$ and $M^{2}=10$ 


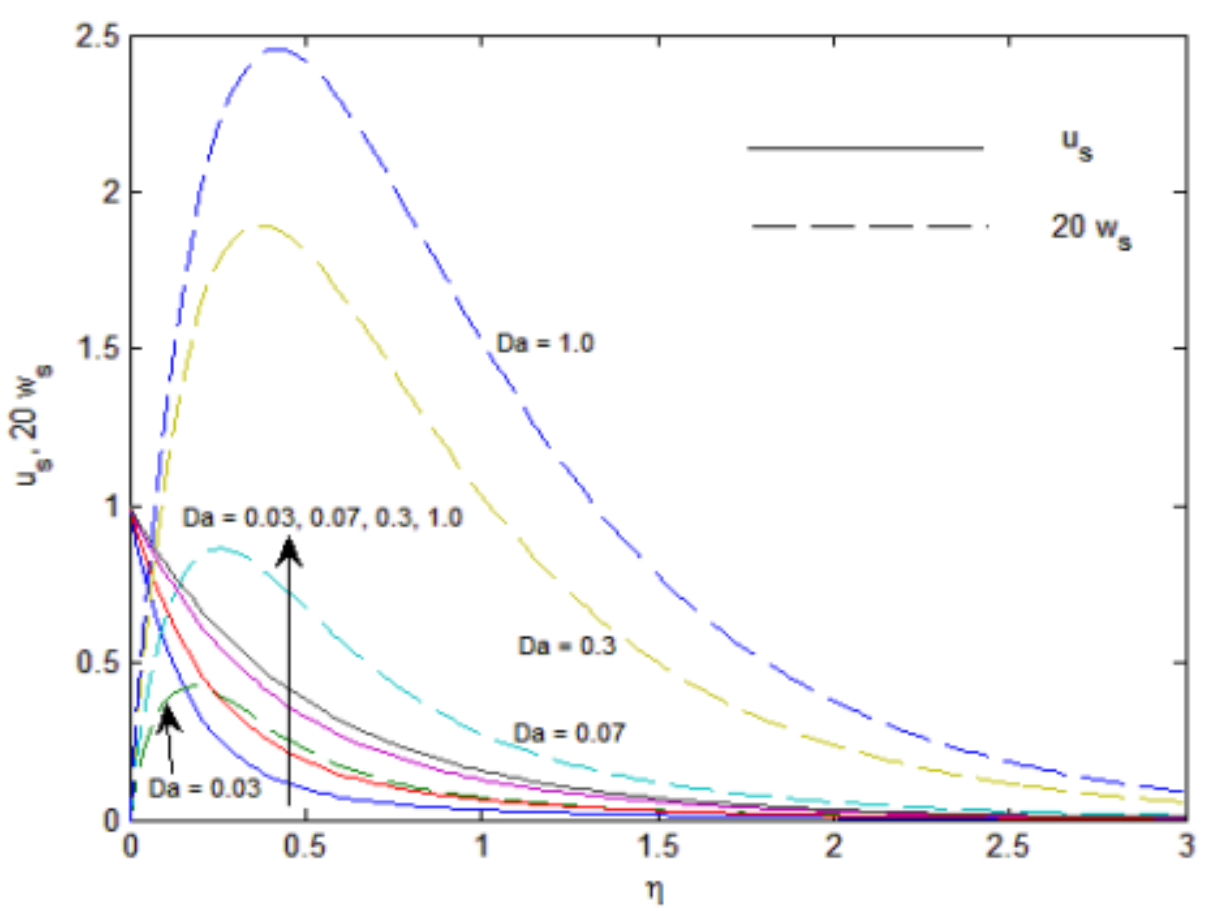

Fig. 6: Steady state velocities for different $D a$ when $m=0.5, G r=5, R a=2$ and $M^{2}=10$

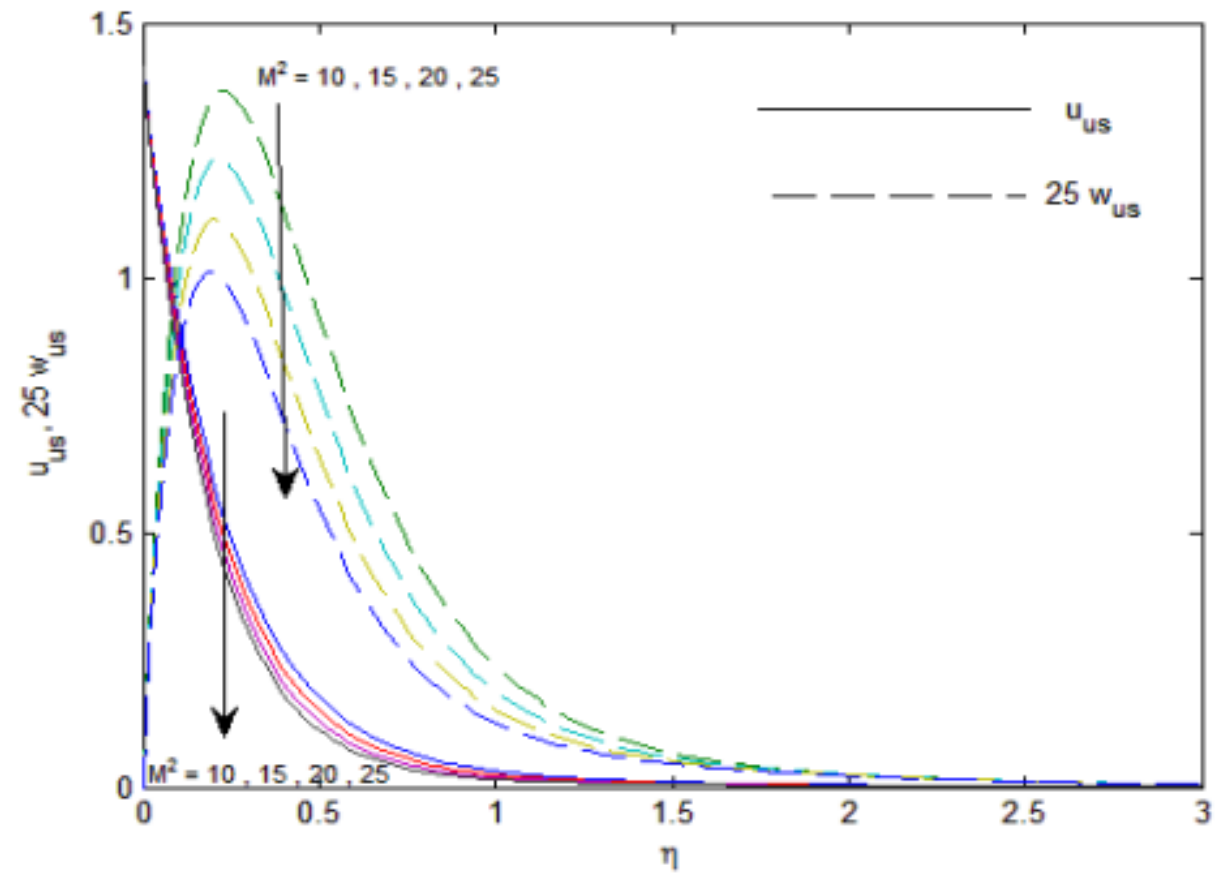

Fig. 7: Unsteady state velocities for different $M^{2}$ when $m=0.5, G r=2 \operatorname{Ra}=2 \operatorname{Pr}=0.03 n=4$,

$$
D a=0.067 \text { and } n \tau=\frac{\pi}{4}
$$




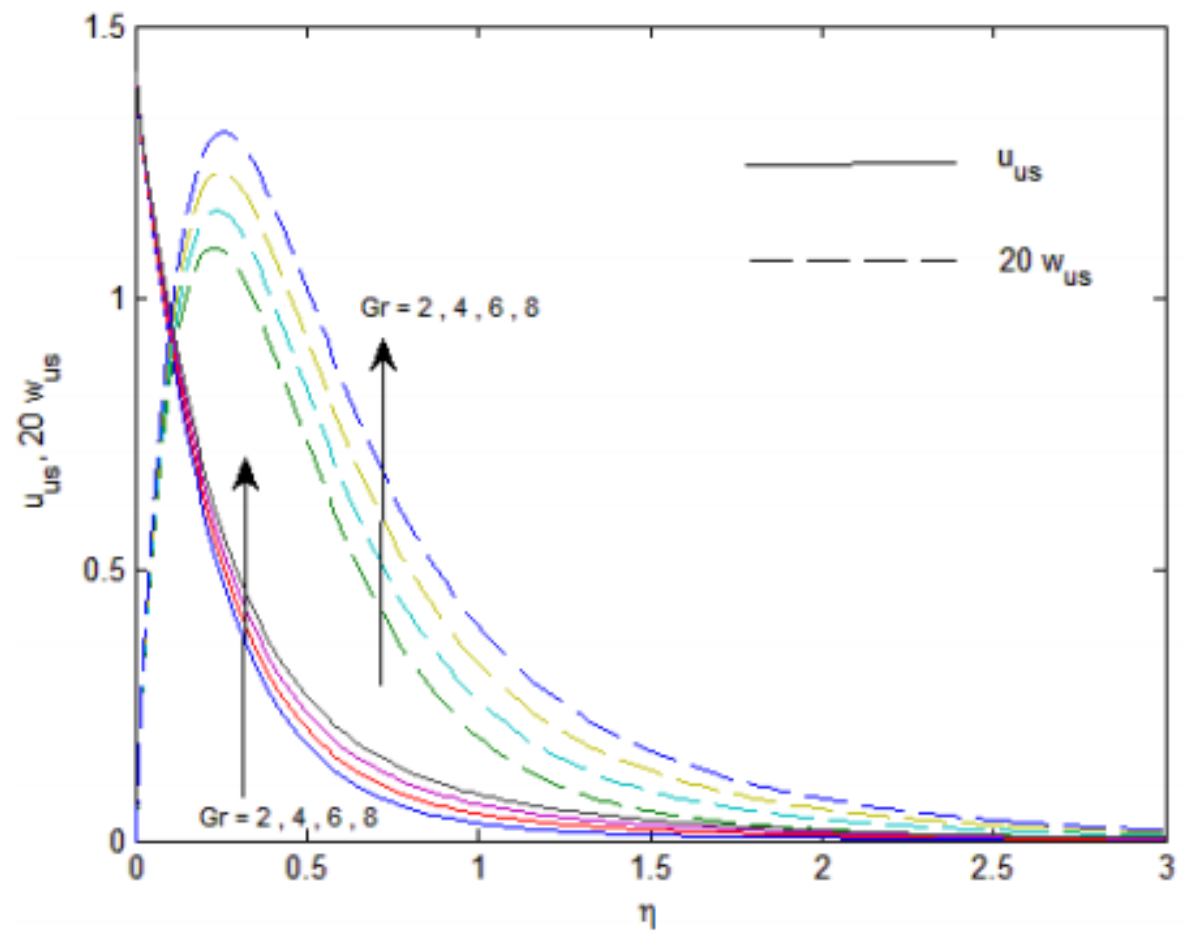

Fig. 8: Unsteady state velocities for different $G r$ when $m=0.5, M^{2}=10, R a=2, \operatorname{Pr}=0.03, n=4$

$$
D a=0.067 \text { and } n \tau=\frac{\pi}{4}
$$

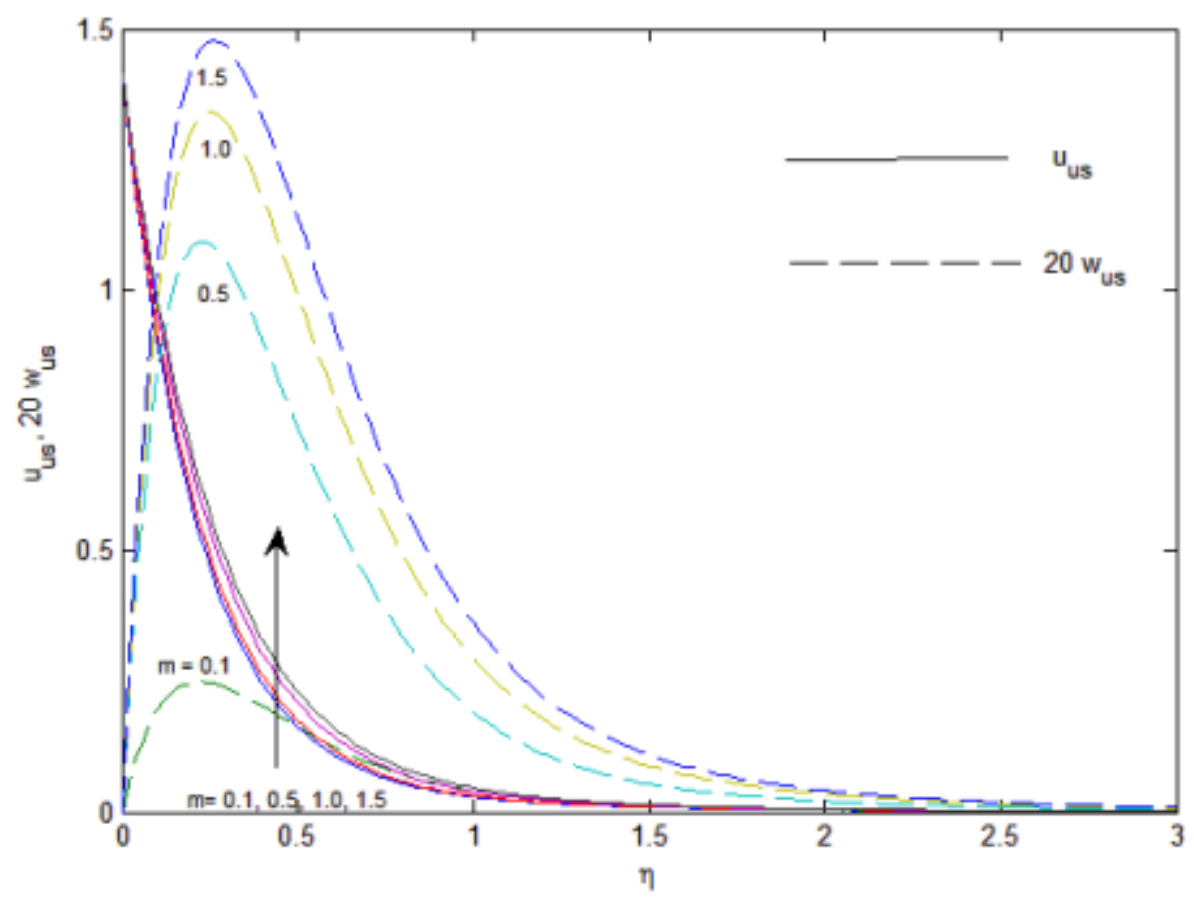

Fig. 9: Unsteady state velocities for different $\mathrm{m}$ when $G r=2, M^{2}=10, R a=2, \operatorname{Pr}=0.03, n=4$,

$$
D a=0.067 \text { and } n \tau=\frac{\pi}{4}
$$




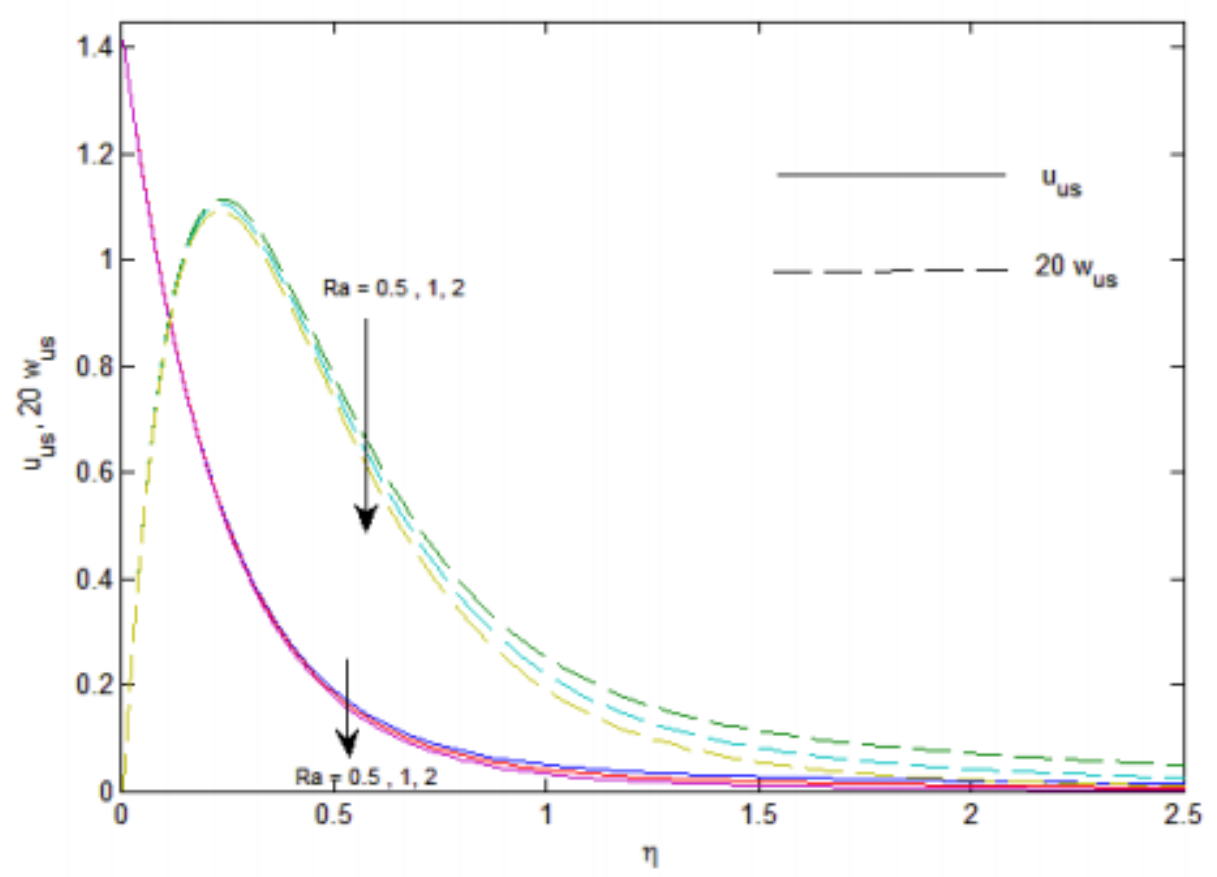

Fig. 10: Unsteady state velocities for different $R a$ when $G r=2, M^{2}=10, m=0.5, P r=0.03$,

$$
n=4, D a=0.067 \text { and } n \tau=\frac{\pi}{4}
$$

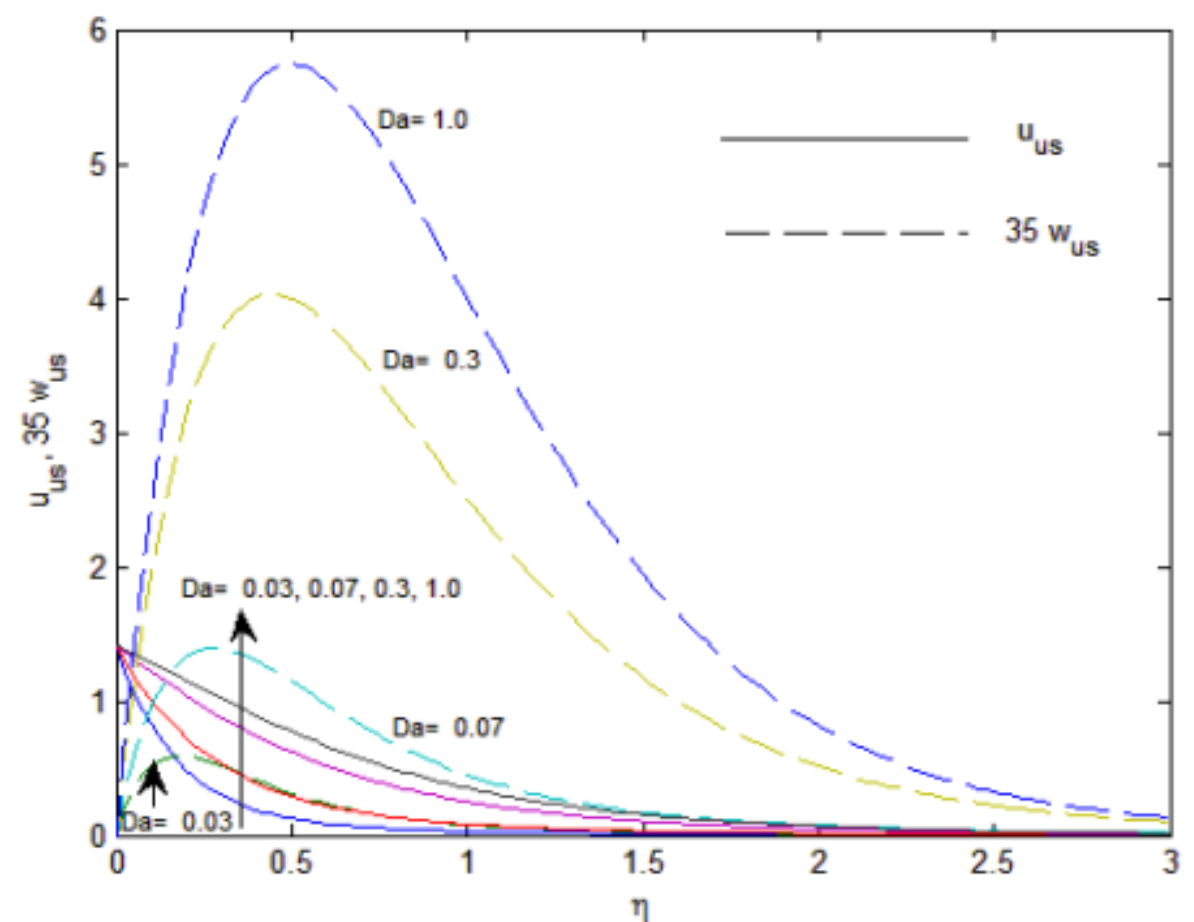

Fig. 11: Unsteady state velocities for different $D a$ when $G r=5, M^{2}=5, m=0.5, P r=0.03$

$$
n=4, \quad R a=2 \text { and } n \tau=\frac{\pi}{4}
$$




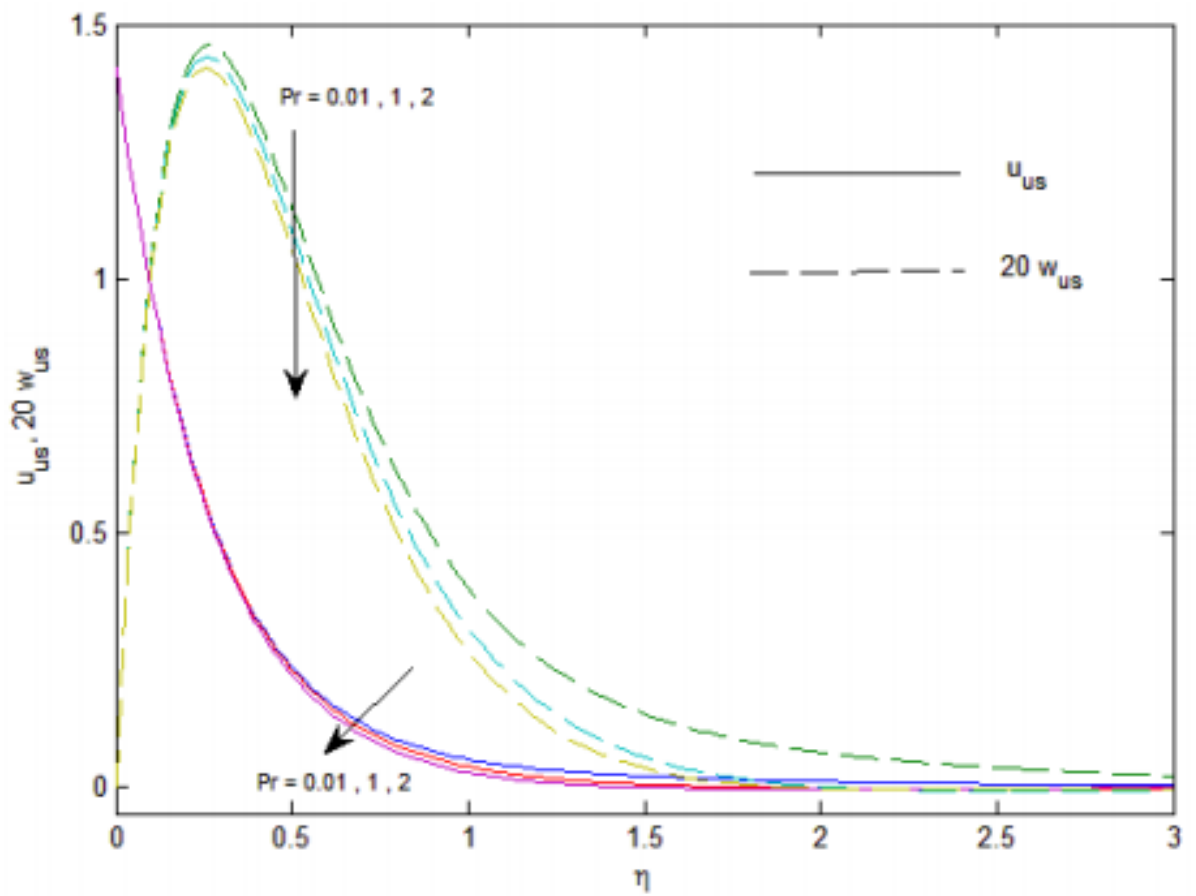

Fig. 12: Unsteady state velocities for different $\operatorname{Pr}$ when $G r=2, M^{2}=10, m=0.5, D a=0.1$,

$$
n=4, R a=1 \text { and } n \tau=\frac{\pi}{4}
$$

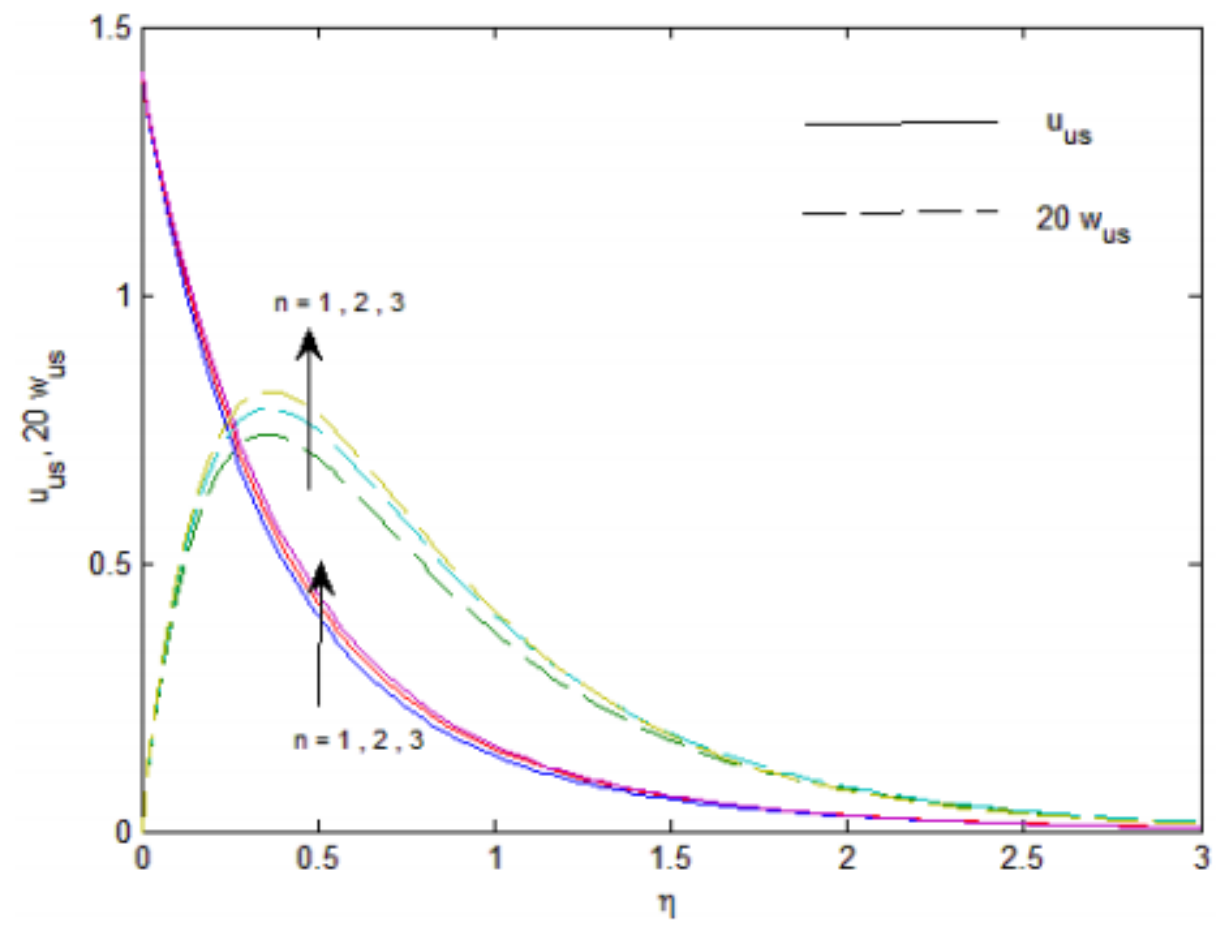

Fig. 13: Unsteady state velocities for different $n$ when $G r=5, M^{2}=2, m=0.5, D a=0.1$,

$$
\operatorname{Pr}=0.3, \quad R a=2 \text { and } n \tau=\frac{\pi}{4}
$$




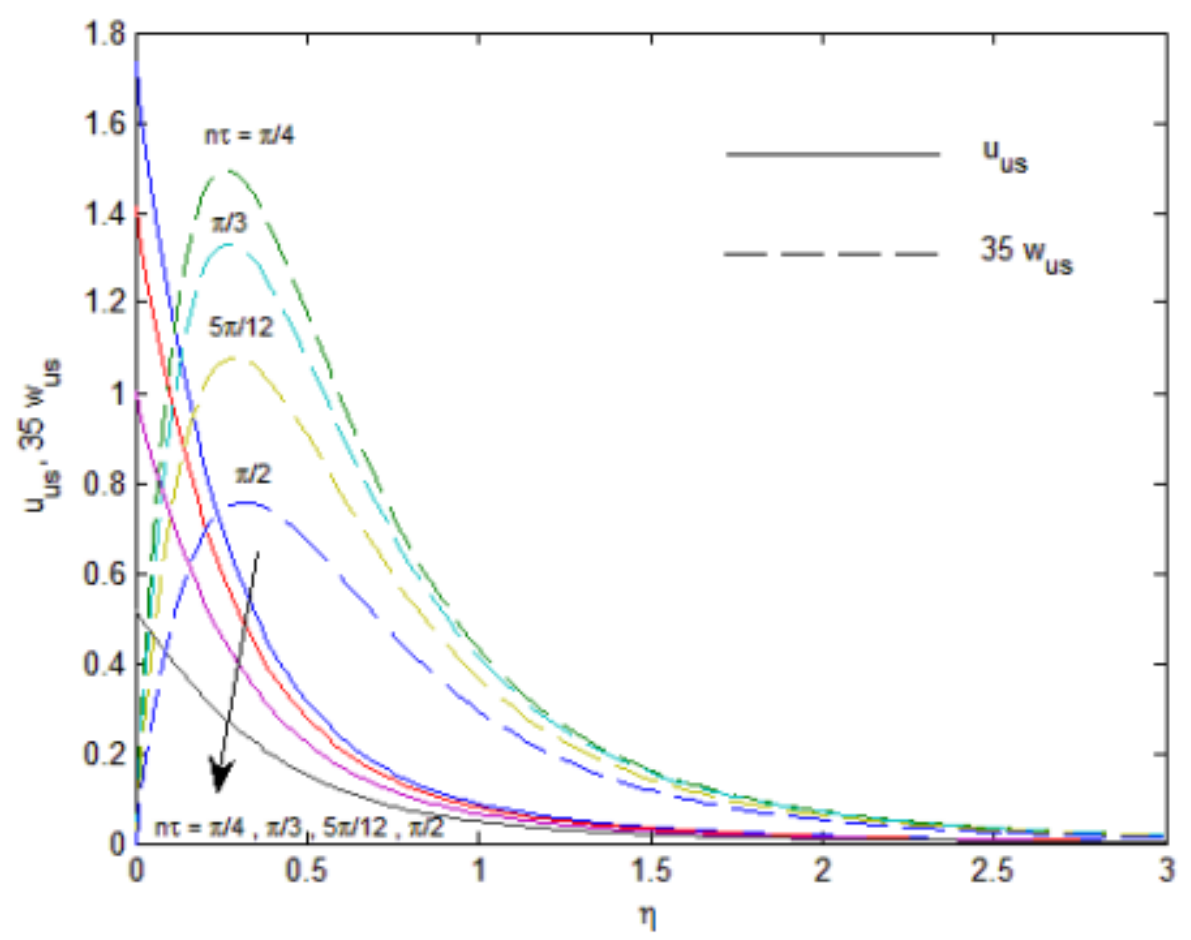

Fig. 14: Unsteady state velocities for different $n \tau$ when $G r=5, M^{2}=5, m=0.5, D a=0.067$, $\operatorname{Pr}=0.03, R a=2$ and $n=4$

The effects of radiation parameter $R a$, Prandtl number $P r$, frequency parameter $n$ and phase angle $n \tau$ on the temperature distribution have been shown in Figs.15-19. It is observed from Fig. 15 and Fig.16 that the fluid temperatures $\theta_{s}$ and $\theta_{u s}$ decrease with an increase in radiation parameter $R a$. This result qualitatively agrees with expectations, since the effect of radiation decrease the rate of energy transport to the fluid, thereby decreasing the temperature of the fluid. Fig.17 shows that the fluid temperature $\theta_{u s}$ decreases with an increase in Prandtl number $\operatorname{Pr}$. Prandtl number $P r$ is the ratio of viscosity to thermal diffusivity. An increase in thermal diffusivity leads to a decrease in Prandtl number. Therefore, thermal diffusion has tendency to reduce the fluid temperature. It is revealed from Fig.18 that an increase in frequency parameter $n$ leads to rise in the fluid temperature distribution $\theta_{u s}$. Fig. 19 shows that the fluid temperature $\theta_{u s}$ decreases with an increase in phase angle $n \tau$.

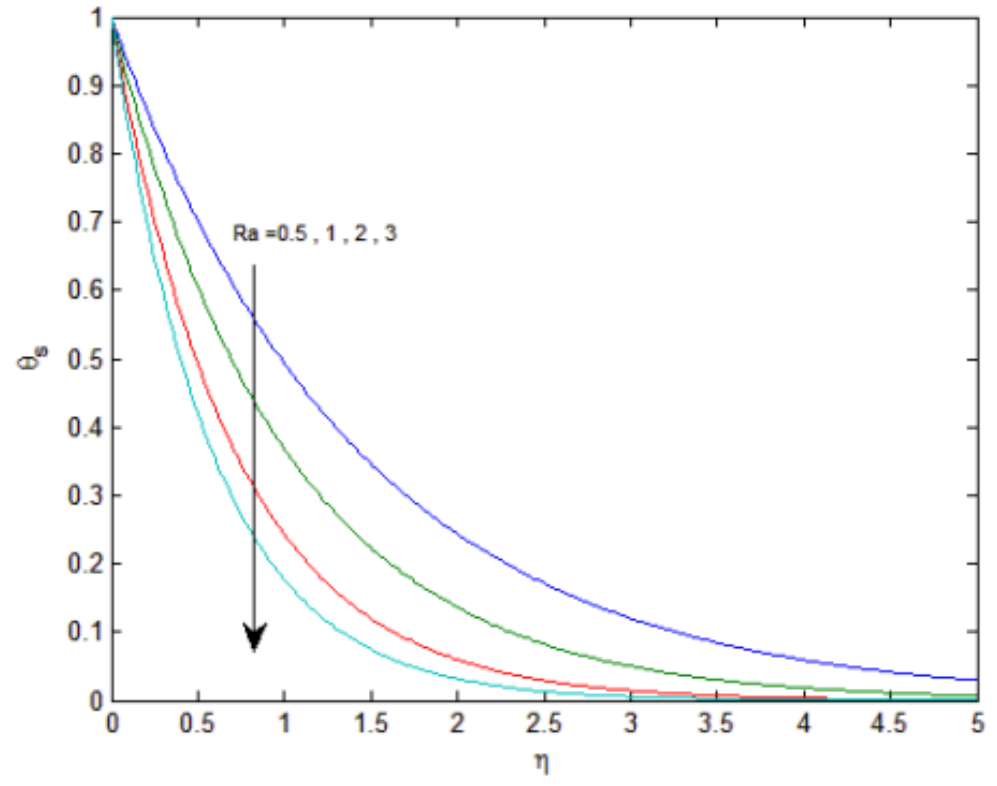

Fig. 15: Steady state temperature for different Ra 


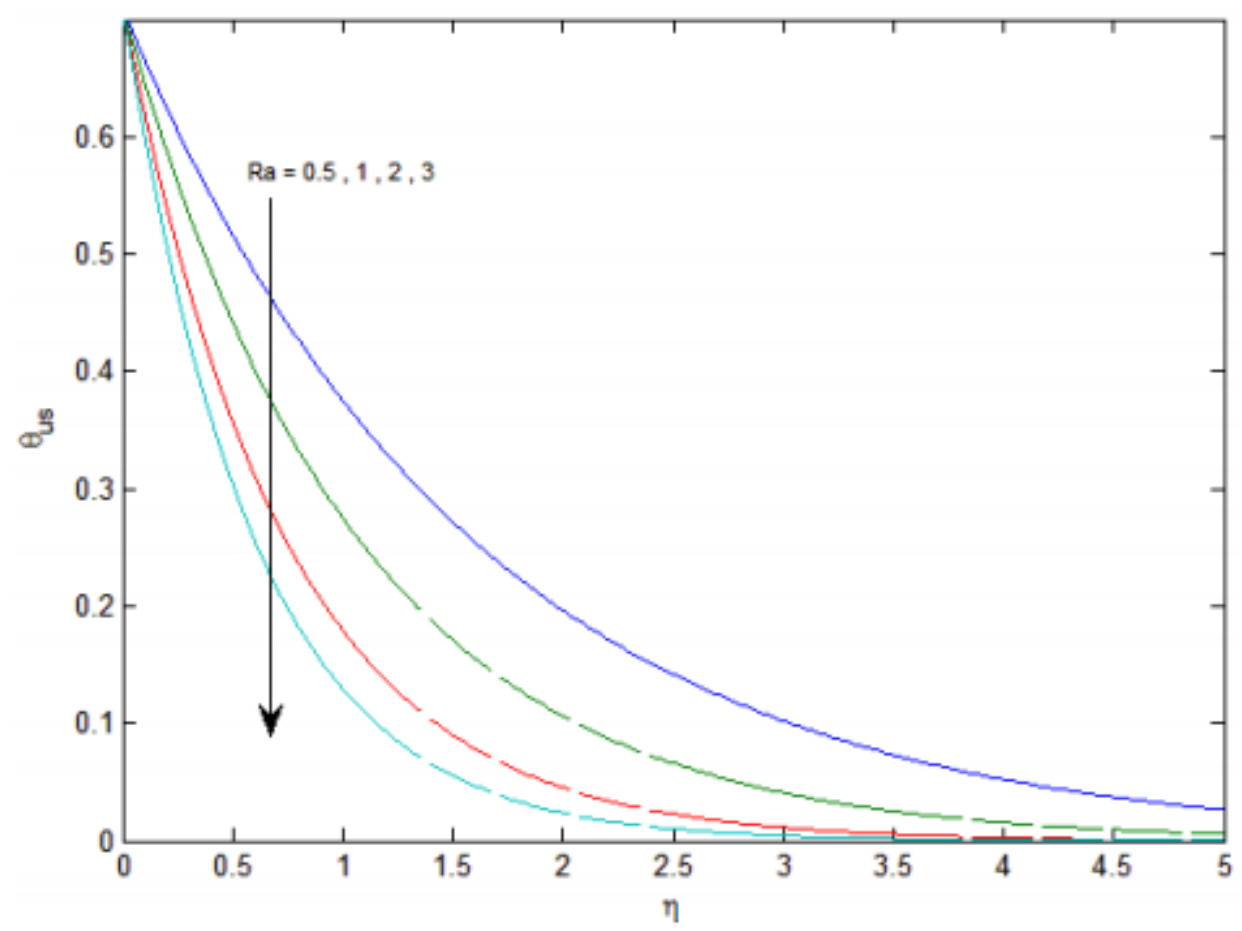

Fig.16: Unsteady state temperature for different $\operatorname{Ra}$ when $\operatorname{Pr}=0.03, n=4$ and $n \tau=\frac{\pi}{4}$

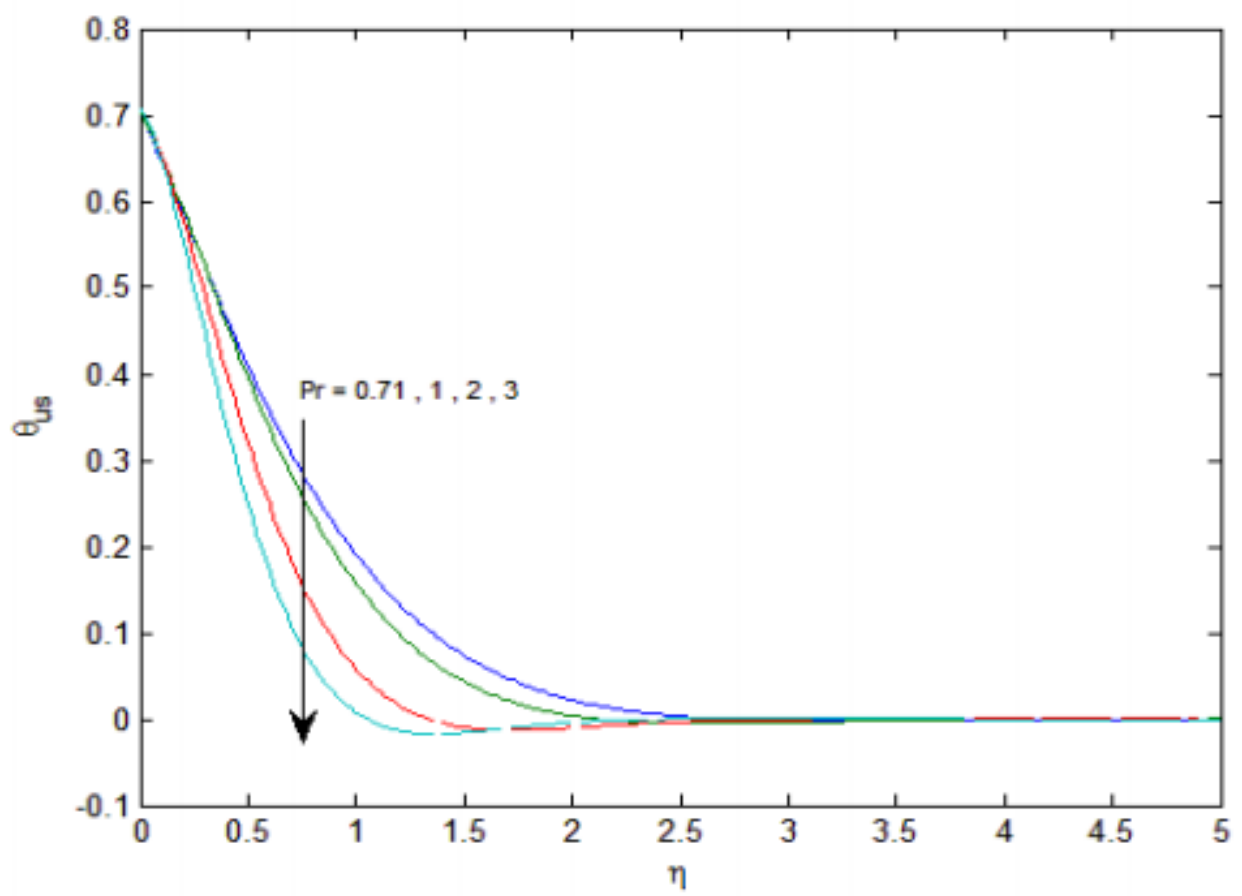

Fig.17: Unsteady state temperature for different $\operatorname{Pr}$ when $R a=2, n=4$ and $n \tau=\frac{\pi}{4}$ 


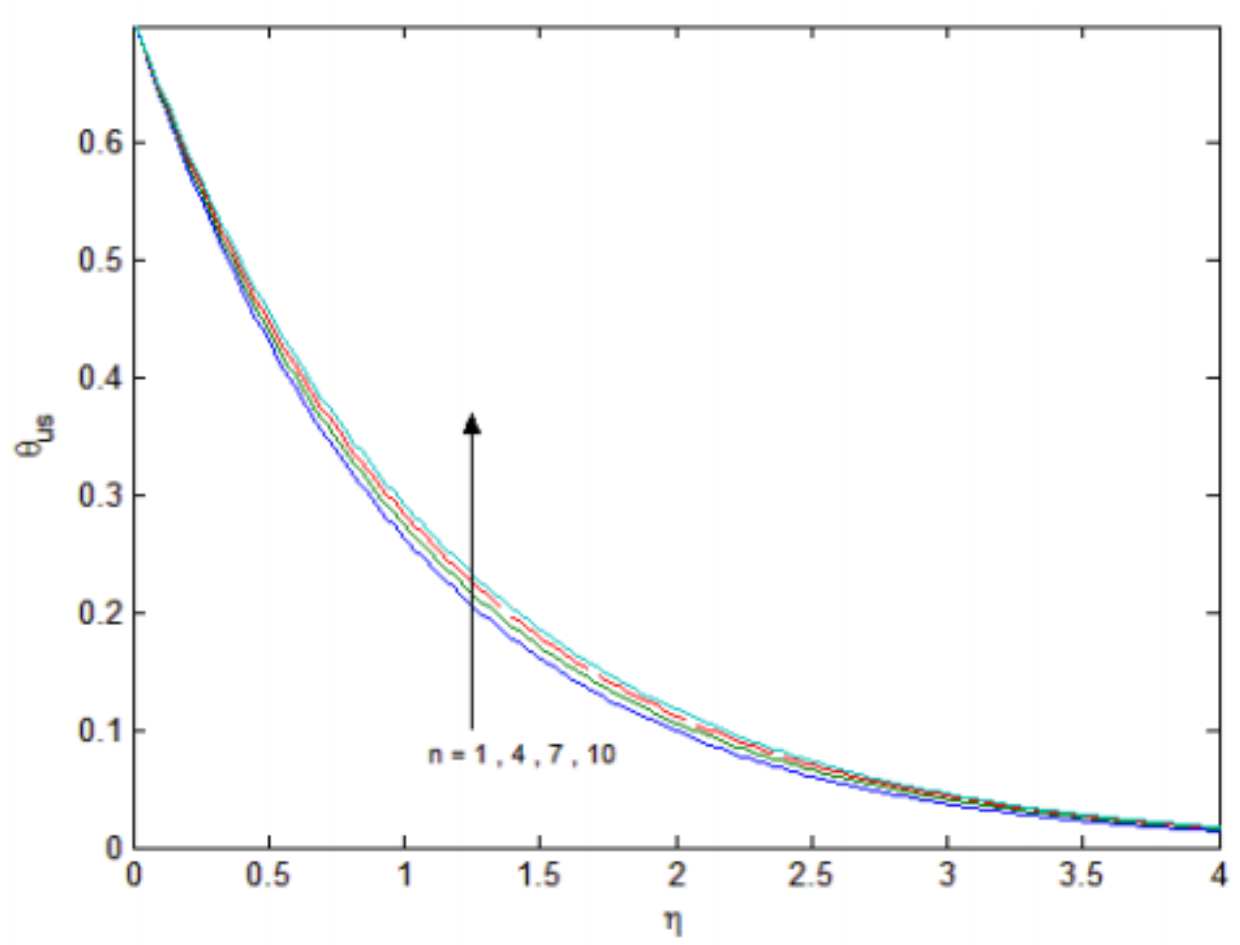

Fig.18: Unsteady state temperature for different $n$ when $\operatorname{Pr}=0.03, \operatorname{Ra}=1$ and $n \tau=\frac{\pi}{4}$

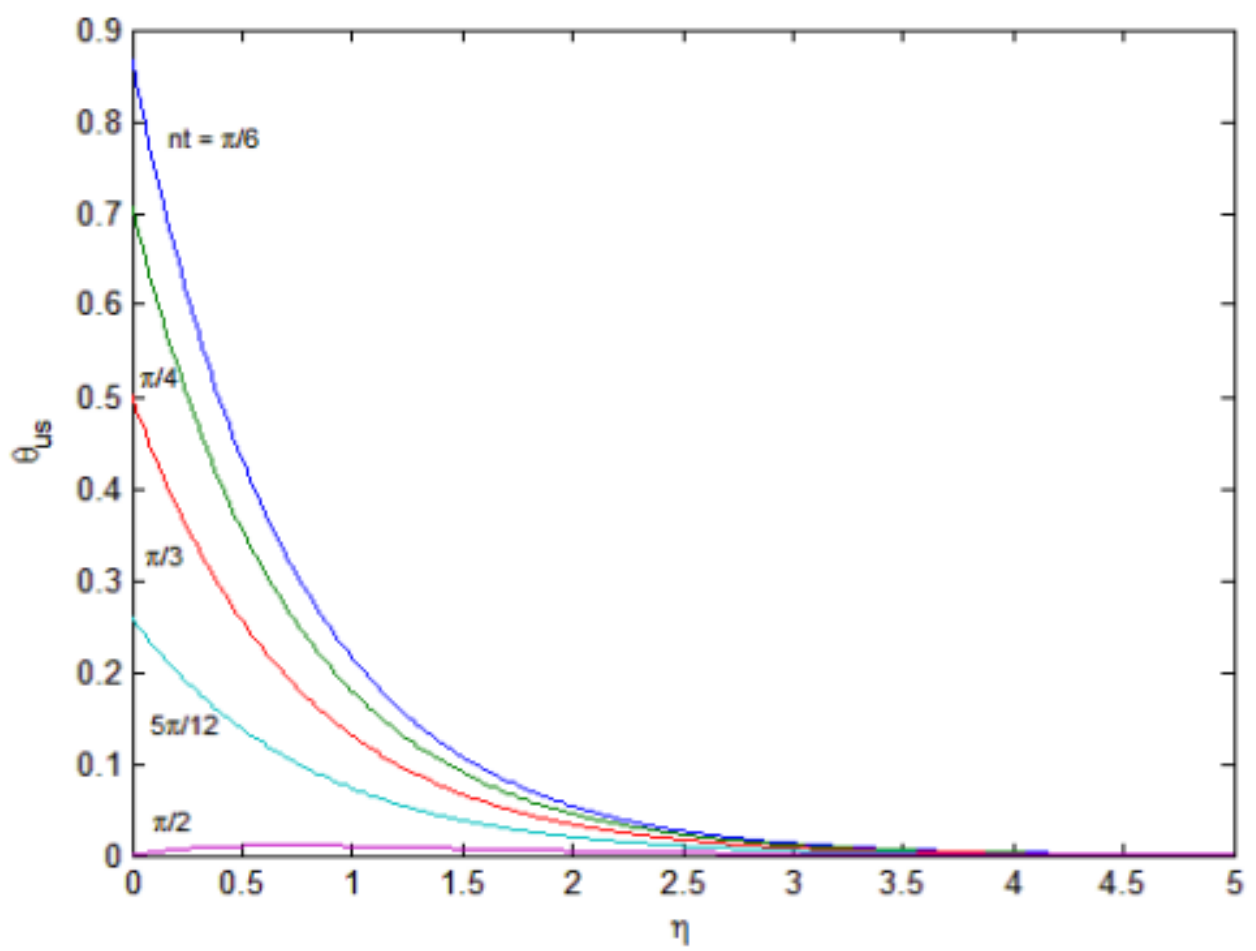

Fig.19: Unsteady state temperature for different $n \tau$ when $\operatorname{Pr}=0.03, n=4$ and $R a=2$

The non-dimensional shear stresses at the plate $(\eta=0)$ due to the steady primary and the secondary flows are respectively

$$
\begin{aligned}
& \left(\tau_{x_{0}}\right)_{s}=\left(\sqrt{R a}-\alpha_{1}\right) x_{1}+\beta_{1} y_{1}-\alpha_{1}, \\
& \left(\tau_{z_{0}}\right)_{s}=\left(\alpha_{1}-\sqrt{R a}\right) y_{1}+\beta_{1} x_{1}+\beta_{1},
\end{aligned}
$$

where $\alpha_{1}$ and $\beta_{1}$ are given by (36) and $x_{1}$ and $y_{1}$ are given by (40). 
The non-dimensional shear stresses at the plate $(\eta=0)$ due to the unsteady primary and the secondary flows are respectively

$$
\begin{aligned}
& \left(\tau_{x_{0}}\right)_{u s}=R_{1} \cos \left(n \tau+\phi_{1}\right), \\
& \left(\tau_{z_{0}}\right)_{u s}=R_{2} \cos \left(n \tau+\phi_{2}\right),
\end{aligned}
$$

Where

$$
\begin{gathered}
R_{1}=\left(p_{1}^{2}+q_{1}^{2}\right)^{\frac{1}{2}}, \tan \phi_{1}=\frac{q_{1}}{p_{1}}, \\
R_{2}=\left(p_{2}^{2}+q_{2}^{2}\right)^{\frac{1}{2}}, \tan \phi_{2}=\frac{q_{2}}{p_{2}}, \\
p_{1}=-\left(\frac{1}{2}+x_{2}\right) \alpha_{2} \mp y_{2} \beta_{2}-\left(\frac{1}{2}+x_{3}\right) \alpha_{3}-y_{3} \beta_{3}+\alpha\left(x_{2}+x_{3}\right)+\beta\left(y_{2}+y_{3}\right), \\
q_{1}=-y_{2} \alpha_{2} \pm\left(\frac{1}{2}+x_{2}\right) \beta_{2}-y_{3} \alpha_{3}+\left(\frac{1}{2}+x_{3}\right) \beta_{3}+\alpha\left(y_{2}+y_{3}\right)-\beta\left(x_{2}+x_{3}\right), \\
p_{2}=y_{2} \alpha_{2} \mp\left(\frac{1}{2}+x_{2}\right) \beta_{2}-y_{3} \alpha_{3}+\left(\frac{1}{2}+x_{3}\right) \beta_{3}-\alpha\left(y_{2}-y_{3}\right)+\beta\left(x_{2}-x_{3}\right), \\
q_{2}=-\left(\frac{1}{2}+x_{2}\right) \alpha_{2} \mp y_{2} \beta_{2}+\left(\frac{1}{2}+x_{3}\right) \alpha_{3}+y_{3} \beta_{3}+\alpha\left(x_{2}-x_{3}\right)+\beta\left(y_{2}-y_{3}\right),
\end{gathered}
$$

where $\alpha, \beta, \alpha_{2}, \beta_{2}, \alpha_{3}$ and $\beta_{3}$ are given by (36) and $x_{2}, y_{2}, x_{3}$ and $y_{3}$ are given by (44).

Equations (48) and (49) show that the shear stress components at the plate $(\eta=0)$ due to the steady flow experience a non-periodic oscillation which depends on the magnetic parameter $M^{2}$, Hall parameter $m$, Grashof number $G r$, Darcy number $D a$ and radiation parameter $R a$. It is seen from equations (50) and (51) that the shear stress components due to the unsteady flow are subjected to periodic oscillations which not only depend on $M^{2}, m, G r, D a$ and $R a$ but also depend on the frequency parameter $n$ as well as Prandtl number $\mathrm{Pr}$.

Numerical results of the shear stresses at the plate $(\eta=0)$ due to the steady flow, the amplitudes and the tangent of phases of shear stresses at the plate $(\eta=0)$ due to the unsteady flow are presented in Tables 1-7 for several values of magnetic parameter $M^{2}$, Hall parameter $m$, the radiation parameter $R a$, Darcy number $D a$, Prandtl number $\operatorname{Pr}$ and frequency parameter $n$ when $G r=5$. Table 1 shows that the absolute value of the shear stress $\left(\tau_{x_{0}}\right)_{s}$ at the plate due to the steady primary flow and the shear stress $\left(\tau_{x_{0}}\right)_{s}$ at the plate due to the steady secondary flow increase with an increase in magnetic parameter

$M^{2}$. It is found from Table 2 that the absolute value of the shear stress $\left(\tau_{x_{0}}\right)_{s}$ at the plate increases while the shear stress $\left(\tau_{x_{0}}\right)_{s}$ at the plate decreases with an increase in radiation parameter $R a$. It is observed from Table 3 that the absolute value of the shear stress $\left(\tau_{x_{0}}\right)_{s}$ at the plate decreases while the shear stress $\left(\tau_{x_{0}}\right)_{s}$ at the plate increases with an increase in Darcy number $D a$. It is revealed from Tables 1,2 and 3 that the absolute value of $\left(\tau_{x_{0}}\right)_{s}$ at the plate decreases whereas the shear stress $\left(\tau_{x_{0}}\right)_{s}$ at the plate increases with an increase in Hall parameter $m$. Table 4 reveals that the amplitude $R_{1}$ of the shear stress at the plate $(\eta=0)$ due to the unsteady primary flow increases with an increase in either frequency parameter $n$ or radiation parameter $R a$ whereas it decreases with an increase in either Darcy number $D a$ or Hall parameter $m$. It is seen from Table 5 that the amplitude $R_{2}$ of the shear stress at the plate $(\eta=0)$ due to the unsteady secondary flow decreases with an increase in eithe frequency parameter $n$ or radiation parameter $R a$ whereas it 
increases with an increase in either Darcy number $D a$ or Hall parameter $m$. Table 6 displays that the tangent of phase $-\tan \phi_{1}$ of the shear stress at the plate $(\eta=0)$ due to the unsteady primary flow decreases with an increase in radiation parameter $R a$ whereas it increases with an increase in either Darcy number $D a$ or frequency parameter $n$ or Hall parameter $m$. It is seen from Table 7 that the tangent of phase $\tan \phi_{2}$ of the shear stress at the plate $(\eta=0)$ due to the unsteady secondary flow decreases with an increase in radiation parameter $R a$ whereas it increases with an increase in either Darcy number $D a$ or frequency parameter $n$ or Hall parameter $m$.

Table 1. Shear stresses $\left(\tau_{x_{0}}\right)_{s}$ and $\left(\tau_{x_{0}}\right)_{s}$ at the plate $(\eta=0)$ when $D a=0.04$ and $R a=1$

\begin{tabular}{|c|c|c|c|c|c|c|c|c|}
\hline & \multicolumn{4}{|c|}{$-\left(\tau_{x_{0}}\right)_{s}$} & \multicolumn{4}{c|}{$\left(\tau_{z_{0}}\right)_{s}$} \\
\hline$m \backslash M^{2}$ & 10 & 15 & 20 & 25 & 10 & 15 & 20 & 25 \\
\hline 0.1 & 5.18464 & 5.63045 & 6.04553 & 6.43534 & 0.09257 & 0.12860 & 0.16037 & 0.18891 \\
0.4 & 5.07268 & 5.47837 & 5.85926 & 6.21909 & 0.32896 & 0.45952 & 0.57533 & 0.67978 \\
0.7 & 4.89731 & 5.23716 & 5.56126 & 5.87097 & 0.46290 & 0.65288 & 0.82331 & 0.97818 \\
1.0 & 4.73014 & 5.00309 & 5.26829 & 5.52541 & 0.50850 & 0.72481 & 0.92169 & 1.10221 \\
\hline
\end{tabular}

Table 2. Shear stresses $\left(\tau_{x_{0}}\right)_{s}$ and $\left(\tau_{z 0}\right)_{s}$ at the plate $(\eta=0)$ when $D a=0.04$ and $M^{2}=10$

\begin{tabular}{|c|c|c|c|c|c|c|c|c|}
\hline & \multicolumn{4}{|c|}{$-\left(\tau_{x_{0}}\right)_{s}$} & \multicolumn{4}{c|}{$\left(\tau_{z_{0}}\right)_{s}$} \\
\hline$m \backslash R a$ & 1 & 2 & 3 & 4 & 1 & 2 & 3 & 4 \\
\hline 0.1 & 5.18464 & 5.22556 & 5.25396 & 5.27612 & 0.09257 & 0.09160 & 0.09097 & 0.09049 \\
0.4 & 5.07268 & 5.11460 & 5.14365 & 5.16632 & 0.32895 & 0.32539 & 0.32305 & 0.32129 \\
0.7 & 4.89731 & 4.94095 & 4.97115 & 4.99468 & 0.46290 & 0.45757 & 0.45408 & 0.45147 \\
1.0 & 4.73014 & 4.77564 & 4.80707 & 4.83151 & 0.50850 & 0.50229 & 0.49823 & 0.49519 \\
\hline
\end{tabular}

Table 3. Shear stresses $\left(\tau_{x_{0}}\right)_{s}$ and $\left(\tau_{z 0}\right)_{s}$ at the plate $(\eta=0)$ when $R a=1$ and $M^{2}=10$

\begin{tabular}{|c|c|c|c|c|c|c|c|c|}
\hline & \multicolumn{4}{|c|}{$-\left(\tau_{x_{0}}\right)_{s}$} & \multicolumn{4}{c|}{$\left(\tau_{z_{0}}\right)_{s}$} \\
\hline$m \backslash D a$ & 0.03 & 0.04 & 0.05 & 0.07 & 0.03 & 0.04 & 0.05 & 0.07 \\
\hline 0.1 & 5.91577 & 5.18464 & 4.69615 & 4.07454 & 0.08184 & 0.09257 & 0.10134 & 0.11500 \\
0.4 & 5.81528 & 5.07268 & 4.57527 & 3.94068 & 0.28968 & 0.32895 & 0.36137 & 0.41243 \\
0.7 & 5.65937 & 4.89731 & 4.38440 & 3.72660 & 0.40488 & 0.46290 & 0.51157 & 0.58975 \\
1.0 & 5.51264 & 4.73014 & 4.20040 & 3.51636 & 0.44163 & 0.50850 & 0.56562 & 0.65943 \\
\hline
\end{tabular}

Table 4. Amplitude $R_{1}$ at the plate $(\eta=0)$ when $M^{2}=10$ and $\operatorname{Pr}=0.03$

\begin{tabular}{|l|c|c|c|c|c|c|c|c|c|}
\hline & \multicolumn{3}{|c|}{$D a$} & \multicolumn{3}{c|}{$n$} & \multicolumn{3}{c|}{$R a$} \\
\hline$m$ & 0.04 & 0.05 & 0.07 & 4 & 5 & 6 & 0.5 & 1 & 2 \\
\hline 0.1 & 5.2117 & 4.7315 & 4.1258 & 5.2117 & 5.2267 & 5.2450 & 5.1813 & 5.2117 & 5.2513 \\
0.3 & 5.1494 & 4.6646 & 4.0524 & 5.1494 & 5.1648 & 5.1835 & 5.1186 & 5.1494 & 5.1895 \\
0.5 & 5.0459 & 4.5531 & 3.9295 & 5.0459 & 5.0621 & 5.0817 & 5.0145 & 5.0459 & 5.0869 \\
0.7 & 4.9328 & 4.4315 & 3.7962 & 4.9328 & 4.9452 & 4.9659 & 4.9006 & 4.9328 & 4.9748 \\
\hline
\end{tabular}


Table 5. Amplitude $R_{2}$ at the plate $(\eta=0)$ when $M^{2}=10$ and $\operatorname{Pr}=0.03$

\begin{tabular}{|l|l|l|l|l|l|l|l|l|l|}
\hline & \multicolumn{2}{|l|}{$\mathrm{Da}$} & \multicolumn{2}{l|}{$n$} & \multicolumn{1}{l|}{$R a$} & \\
\hline$m$ & 0.04 & 0.05 & 0.07 & 4 & 5 & 6 & 0.5 & 1 & 2 \\
\hline .1 & 0.09216 & 0.10071 & 0.11390 & 0.09216 & 0.09193 & 0.09165 & 0.09291 & 0.09216 & 0.09122 \\
0.3 & 0.25905 & 0.28363 & 0.32171 & 0.25905 & 0.25839 & 0.25759 & 0.26121 & 0.25905 & 0.25637 \\
0.5 & 0.38362 & 0.42137 & 0.48046 & 0.38362 & 0.38258 & 0.38133 & 0.38694 & 0.38362 & 0.37952 \\
0.7 & 0.40872 & 0.45359 & 0.52596 & 0.40872 & 0.45914 & 0.45752 & 0.41273 & 0.40872 & 0.40392 \\
\hline
\end{tabular}

Table 6. Tangent of phase $-\tan \phi_{1}$ at the plate $(\eta=0)$ when $M^{2}=10$ and $\operatorname{Pr}=0.03$

\begin{tabular}{|c|c|c|c|c|c|c|c|c|c|}
\hline & \multicolumn{3}{|c|}{$D a$} & \multicolumn{3}{c|}{$n$} & \multicolumn{3}{c|}{$R a$} \\
\hline$m$ & 0.04 & 0.05 & 0.07 & 4 & 5 & 6 & 0.5 & 1 & 2 \\
\hline .1 & 0.07296 & 0.08806 & 0.11479 & 0.07296 & 0.09096 & 0.10879 & 0.07464 & 0.07296 & 0.07124 \\
0.3 & 0.07456 & 0.09030 & 0.11835 & 0.07456 & 0.09294 & 0.11115 & 0.07630 & 0.07456 & 0.07277 \\
0.5 & 0.07737 & 0.09429 & 0.12484 & 0.07737 & 0.09642 & 0.11530 & 0.07922 & 0.07737 & 0.07545 \\
0.7 & 0.09138 & 0.11163 & 0.14842 & 0.09138 & 0.10071 & 0.12039 & 0.09348 & 0.09138 & 0.08916 \\
\hline
\end{tabular}

Table 7. Tangent of phase $\tan \phi_{2}$ at the plate $(\eta=0)$ when $M^{2}=10$ and $\operatorname{Pr}=0.03$

\begin{tabular}{|c|c|c|c|c|c|c|c|c|c|}
\hline & \multicolumn{3}{|c|}{$D a$} & \multicolumn{3}{c|}{$n$} & \multicolumn{3}{c|}{$R a$} \\
\hline$m$ & 0.04 & 0.05 & 0.07 & 4 & 5 & 6 & 0.5 & 1 & 2 \\
\hline 0.1 & 0.06794 & 0.08045 & 0.10147 & 0.06794 & 0.08474 & 0.10141 & 0.07006 & 0.06794 & 0.06591 \\
0.3 & 0.06929 & 0.08227 & 0.10418 & 0.06929 & 0.08641 & 0.10341 & 0.07147 & 0.06929 & 0.06719 \\
0.5 & 0.07165 & 0.08548 & 0.10905 & 0.07165 & 0.08934 & 0.10690 & 0.07394 & 0.07165 & 0.06944 \\
0.7 & 0.08336 & 0.09932 & 0.12636 & 0.08336 & 0.09292 & 0.11116 & 0.08607 & 0.08336 & 0.08070 \\
\hline
\end{tabular}

The rate of heat transfer at the plate $(\eta=0)$ due to the steady flow is

$$
-\left(\frac{d \theta_{s}}{d \eta}\right)_{\eta=0}=\sqrt{R a} .
$$

The rate of heat transfer at the plate $(\eta=0)$ due to the unsteady flow is

$$
-\left(\frac{d \theta_{u s}}{d \eta}\right)_{\eta=0}=-R_{3} \cos \left(n \tau+\phi_{3}\right),
$$

Where $R_{3}=\left(\alpha^{2}+\beta^{2}\right)^{\frac{1}{2}}$ is the amplitude and $\tan \phi_{3}=-\beta / \alpha$ is the tangent of phase of the rate of heat transfer at the plate $(\eta=0)$ due to the unsteady flow, $\alpha$ and $\beta$ are given by (36).

The numerical values of the amplitudes and tangent of phases of the rate of heat transfer at the plate $(\eta=0)$ due to the unsteady flow are entered in the Tables 8 and 9 for several values of Prandtl number $P r$, radiation parameter $R a$ and frequency parameter $n$. Table 8 shows that the amplitude $R_{3}$ of the rate of heat transfer at the plate $(\eta=0)$ due to the unsteady flow increases with an increase in either $\operatorname{Pr}$ or $R a$ or $n$. It is found from Table 9 that the tangent of phase $-\tan \phi_{3}$ of the rate of heat transfer at the plate $(\eta=0)$ due to the unsteady flow increases with an increase in either Prandtl number $\operatorname{Pr}$ or frequency parameter $n$ whereas it decreases with an increase in radiation parameter $R a$. 
Table 8. Amplitude $R_{3}$ of the rate of heat transfer at the plate $(\eta=0)$

\begin{tabular}{|c|c|c|c|c|c|c|}
\hline & \multicolumn{3}{|c|}{$P r$} & \multicolumn{3}{c|}{$R a$} \\
\hline$n$ & 0.2 & 0.4 & 0.6 & 1 & 2 & 3 \\
\hline 1 & 1.00985 & 1.03780 & 1.07990 & 1.00022 & 1.41429 & 1.73209 \\
2 & 1.03780 & 1.13165 & 1.24982 & 1.00090 & 1.41453 & 1.73222 \\
3 & 1.07990 & 1.24982 & 1.43497 & 1.00202 & 1.41493 & 1.73244 \\
4 & 1.13165 & 1.37361 & 1.61245 & 1.00358 & 1.41548 & 1.73274 \\
\hline
\end{tabular}

Table 9. Tangent of phase $-\tan \phi_{3}$ of the rate of heat transfer at the plate $(\eta=0)$

\begin{tabular}{|c|c|c|c|c|c|c|}
\hline & \multicolumn{3}{|c|}{$P r$} & \multicolumn{3}{c|}{$R a$} \\
\hline$n$ & 0.2 & 0.4 & 0.6 & 1 & 2 & 3 \\
\hline 1 & 0.09902 & 0.19258 & 0.27698 & 0.01500 & 0.00750 & 0.00500 \\
2 & 0.19258 & 0.35078 & 0.46837 & 0.02997 & 0.01500 & 0.01000 \\
3 & 0.27698 & 0.46837 & 0.58840 & 0.04491 & 0.02249 & 0.01500 \\
4 & 0.35078 & 0.55425 & 0.66667 & 0.05979 & 0.02997 & 0.01999 \\
\hline
\end{tabular}

\section{Conclusion}

The combined effects of Hall currents and radiation on an unsteady MHD free convective flow of a viscous incompressible electrically conducting fluid past a vertical flat plate with oscillatory plate temperature embedded in porous medium have been investigated. Thermal radiation retards the steady as well as the unsteady velocity components whereas buoyancy force and Hall currents accelerate the velocity components. The porosity of the medium has an accelerating effect on the velocity field. Thermal radiation leads to fall the fluid temperature for both the steady as well as unsteady flow. The fluid temperature decreases with an increase in Prandtl number. The tangent of phase $-\tan \phi_{1}$ of the shear stress at the plate due to the unsteady primary flow increases with an increase in either Darcy number $D a$ or Hall parameter $m$. Further, the amplitudes and tangent of phases of the rate of heat transfer at the plate due to the unsteady flow increase with an increase in frequency parameter.

\section{References}

[1] Takhar, H.S. and Ram, P.C.(1991): Free convection in hydromagnetic flows of a viscous heatgenerating fluid with wall temperature oscillation and Hall currents, Astrophysics and Space Science, 183, 193-198.

[2] Sattar, M. A. and Hossain, M. M. (1992): Unsteady hydromagnetic free convection flow with Hall current and mass transfer along an accelerated porous plate with time dependent temperature and concentration, Can. J. Phys., 70, 369-375.

[3] Alagoa, K.D., Tay, G. and Abbey, T.M. (1998): Radiative and free convective effects of MHD flow through a porous medium between infinite parallel plates with time-dependent suction, Astrophysics and Space Science, 260(4), 455-468.

[4] Kinyanjui, M., Chartuverdi, N., Uppal, S.M. (2001): Magnetohydrodynamic free convection heat and mass transfer of a heat generating fluid past an impulsively started infinite vertical porous plate with Hall current and radiation absorption, Energy Convers. Manage., 42(8), 917-931.

[5] Hayat, T., Wang, Y., Hutter, K. (2004): Hall effects on the unsteady hydromagnetic oscillatory flow of a second-grade fluid, Int. J. Non-Linear Mech., 39(6), 1027-1037.

[6] Nield, D. A. and Bejan, A. (2006): Convection in porous media, Springer, New York, pp. 9497. 
[7] Samad, M.A. and Rahman, M.M. (2006): Thermal radiation interaction with unsteady MHD flow past a vertical porous plate immersed in a porous medium, J. Naval Arch. Marine Eng., 3, 714.

[8] Mbeledogu, I. U. and Ogulu, A. (2007): Heat and mass transfer of an unsteady MHD natural convection flow of a rotating fluid past a vertical porous flat plate in the presence of radiative heat transfer, Int. J. Heat Mass Transfer, 50, 1902.

[9] Beg, O.A. and Ghosh, S.K. (2010): Analytical study of magnetohydrodynamic radiationconvection with surface temperature oscillation and secondary flow effects, Int. J. Appl. Math. Mech., 6(6) 1-22.

[10] Shateyi, S., Motsa, S.S. and Sibanda, P. (2010): The effects of thermal radiation, Hall currents, Soret and Dufour on MHD flow by mixed convection over a vertical surface in porous media, Math. Prob. Engin., 2010, 20, doi:10.1155/2010/627475.

[11] Das, S., Jana, M. and Jana, R. N. (2011): Radiation effect on natural convection near a vertical plate embedded in porous medium with ramped wall temperature, Op. J. Flu. Dynamics, 1, 1-11.

[12] Das, S., Jana, M. and Jana, R. N. (2011): Effects of radiation on free convection flow in a vertical channel embedded in porous media, Int. J. Comp. Appl., 35(6), 38-44.

[13] Veera. Krishna. M. and Sujatha. P. (2011): MHD free and forced convection flow of newtonian fluid through a porous medium in an infinite vertical plate in presence of thermal radiation heat transfer and surface temperature oscillation, Int. J. Phy. and Math. Sc., 1(1), 33-52.

[14] Cowling, T. G. (1957): Magnetohydrodynamics, New York, Interscience, pp.101.

[15] Siegel, R. and Howell, J.R. (1993): Thermal Radiation Heat Transfer, New York: Hemisphere, USA 